\title{
MONITORING OF CHLORINE CONCENTRATION IN DRINKING WATER DISTRIBUTION SYSTEMS USING AN INTERVAL ESTIMATOR
}

\author{
RAFAŁ ŁANGOWSKI*, MIETEK A. BRDYS *,** \\ * Department of Automatic Control, Gdańsk University of Technology \\ ul. G. Narutowicza 11/12, 80-952 Gdańsk, Poland, \\ e-mail: rlangow@ely.pg.gda.pl \\ ** Department of Electronic, Electrical and Computer Engineering \\ School of Engineering, University of Birmingham, Birmingham B15 2TT, UK \\ e-mail: m.brdys@bham.ac.uk
}

\begin{abstract}
This paper describes the design of an interval observer for the estimation of unmeasured quality state variables in drinking water distribution systems. The estimator utilizes a set bounded model of uncertainty to produce robust interval bounds on the estimated state variables of the water quality. The bounds are generated by solving two differential equations. Hence the numerical efficiency is sufficient for on-line monitoring of the water quality. The observer is applied to an exemplary water network and its performance is validated by simulations.
\end{abstract}

Keywords: estimators, bounding methods, modelling dynamics, water quality

\section{Introduction}

Information about state variables is important for safe and efficient process operation. Monitoring the system in question provides this kind of knowledge. Unfortunately, all process state variables can rarely be directly measured. This is because of the lack of suitable sensors or due to their high cost. Therefore, the missing information about states has to be recovered by employing their estimate that is based on other hard measurements and a mathematical model of the process. The modelling involves also modelling uncertainty. There are several approaches to uncertainty modelling. A set-membership approach is an attractive option (Brdys and Kang, 1994). Optimisation based set-membership algorithms for set bounded estimation of the quantity and integrated quantity and quality in drinking water distribution systems are presented in (Brdys and Chen, 1995; Duzinkiewicz, 2006) and (Brdys and Chen, 1996), respectively. Unfortunately, due to the necessity of solving highly non-linear and non-convex optimisation problems during the estimation process, such estimation methods are time-consuming, and thus not always applicable to on-line estimation. Hence, the paper proposes an alternative estimation method that further develops an observer known as an interval observer (Gouzé et al., 2000) so that it can now be realistically applied to the estimation of the water quality in drinking water distribution systems (DWDSs).

The paper is organized as follows: A physical quality model of a DWDS and a model for the estimation purposes are presented in Section 2. The quality estimation problem is analyzed in Section 3 and the estimation problem is quantitatively formulated. Next, the interval observer is derived in Section 4 and its computational efficiency is assessed in Section 5. In Section 6, the proposed observer is applied to quality estimation in an exemplary DWDS and its performance is validated by simulations. Finally, the conclusions are drawn and future work is presented.

\section{Presentation of Drinking Water Distribution Systems}

The main objective for a DWDS is to meet demand on water of required quality for all industrial and domestic consumers (Brdys and Ulanicki, 1994). On-line information about the state of the DWDS for efficient operation of the DWDS is needed. Both the water quality monitoring system and the water quantity monitoring system deliver this kind of information. In this paper, the water quality mon- 
itoring system is considered. Measurements of the water quality are composed of two types: laboratory and on-line. Bacteriology measurements are among typical laboratory measurements in DWDSs (e.g., the number of coli bacteria).

Due to obvious reasons, this kind of measurements cannot be utilized on-line. At present, the main on-line quality measurement in a DWDS is free chlorine concentration. The water quality is determined by quality factors. There are many such factors. Disinfectant concentration is one of the most popular ones. Chlorine is one of the mostly utilized disinfectants. In this paper, the water quality is determined by free chlorine concentration. Hence, free chlorine concentration measurements in the network nodes are most important for gathering knowledge about the quality state of the DWDS. Clearly, it is impossible to locate chlorine concentration sensors at all nodes of the DWDS. Therefore, achieving complete knowledge about the DWDS state requires state estimation.

Because chlorine reacts with organic and nonorganic matter in water, the chlorine concentration decreases in time (chlorine decay) (Males et al., 1988; Rossman et al., 1994; Clark et al., 1995; Boccelli et al., 2003). If the concentration is too low, pathogenic bacteria can grow in water and this growth can even lead to a bacteriological instability. Strong chlorination performed at water treatment stations and within the network by booster stations can recover safe water. However, this activity can increase the concentration of disinfections by-products (DBPs) being chemical compounds that are produced by the reaction of free chlorine with organic components in water. Trihalomethanes (THMs) are common harmful DBPs. A THM formation model can be found in (Boccelli et al., 2003). THMs are formed in a small amount but their cancerous character must be taken into account at least by maintaining the free chlorine concentration below certain upper limit.

2.1. Quality Model. A DWDS quality model is necessary for water quality estimation purposes. The basic quality model requires the following assumptions to be met:

(1) The water distribution network is composed of water sources, pipes and nodes.

(2) Flow directions are constant over considered time horizon.

(3) All hydraulic quantities (i.e., flow rates, linear water flow velocities, etc.) are available.

(4) Concentrations of chlorine at the external water sources are known.

The water quality model considers changes in the chlorine concentration at nodes and along pipes. Note that the magnitudes and directions of water flows determine the structure of the water quality model. Hence, under the assumption (2), the main structure of the water quality model is constant over the time horizon considered. This assumption is introduced due to the clarity of presentation. It will be shown how it can be altered in order to allow for strong variations in the hydraulic quantities over considered time horizon. Additionally, tanks can easily be introduced into the model.

Physical models of chlorine decay during the water transfer through the network elements can be found in many papers. Models of chlorine decay at pipes can be found in (Males et al., 1988; Rossman et al., 1994; Clark et al., 1995; Park and Kuo, 1999). It was shown (Al-Omari and Chaudhry, 2001) that for turbulent flows the diffusive transport can be disregarded and the equation describing the advection chlorine transport with first-order decay for pipes is as follows:

$$
\frac{\partial C_{A}(z, t)}{\partial t}+v(z, t) \frac{\partial C_{A}(z, t)}{\partial z}-k_{A} C_{A}(z, t)=0
$$

subject to the initial and boundary conditions

$$
\begin{aligned}
& C_{A}(z, 0)=C_{0, t}(z), \\
& C_{A}(0, t)=C_{0, z}(t),
\end{aligned}
$$

respectively, where $t$ is the time instant over the hydraulic step period, $C_{A}(z, t)$ stands for the chlorine concentration in pipe at the instant $t$ at the distance of $z$ from the initial node, $v(z, t)$ signifies the linear water flow velocity, and $k_{A}$ denotes the bulk reaction rate coefficient (it is negative due to the chlorine concentration decay in time).

Remark 1. Notice that the linear water flow velocity and the flow rate are the same at a given pipe, i.e., $v(z, t)=$ $v(t)$. This is because of the assumption of water incompressibility and because the pipes are of the pressure type.

When modelling the water quality at the network nodes, we should distinguish between two types of nodes: quality controlled nodes (Boulos et al., 2004) and uncontrolled quality nodes. The chlorine is directly dosed into the controlled quality nodes in order to maintain the required chlorine concentration, which is prescribed by the quality controller (Duzinkiewicz et al., 2005). A typical arrangement involves a booster station and a PI control loop around the node. The control transients are fast enough to consider the node as having the chlorine concentration independent on the pipe flows entering the nodal junction. Without any loss of generality, such nodes are not considered in the paper. In turn, the quality in the uncontrolled nodes depends on the chlorine concentrations at the pipes supplying water into these nodes. Indeed, the following holds:

$$
\begin{aligned}
\sum_{l \in W E n(t)} Q_{l}(t) C_{A, l}(t) & +\sum_{d \in D n(t)} Q_{d}(t) C_{A, d}(t) \\
& -\sum_{q \in W Y n(t)} Q_{q}(t) C_{A, q}(t)=0,
\end{aligned}
$$


where $W E n(t)$ is the set of pipes supplying water to the node $n$ at the instant $t, W Y n(t)$ denotes the set of pipes draining water from the node $n$ at the instant $t$ into the network, $D n(t)$ stands for the set of pipes supplying water from external sources to the node $n$ at the instant $t, Q_{l}(t)$, $Q_{d}(t), Q_{q}(t)$ are the corresponding flow rates, $C_{A, l}(t)$ and $C_{A, d}(t)$ are the corresponding chlorine concentrations into the node $n$ at the instant $t$, and $C_{A, q}(t)$ is the corresponding chlorine concentration out of the node $n$ at $t$.

Assuming instantaneous and complete mixing of the chlorine at the node $n$, we get

$$
C_{A, q}(t)=C_{A, \text { out }, n}(t),
$$

where $C_{A, \text { out }, n}(t)$ is the resulting chlorine concentration at the node $n$ at the instant $t$.

$$
\begin{aligned}
& \sum_{l \in W E n(t)}^{\text {Combining (3) and (2) gives }} Q_{l}(t) C_{A, l}(t)+\sum_{d \in D n(t)} Q_{d}(t) C_{A, d}(t) \\
& -C_{A, \text { out }, n}(t) \sum_{q \in W Y n(t)} Q_{q}(t)=0 .
\end{aligned}
$$

The mass balance law yields

$$
\sum_{l \in W E n(t)} Q_{l}(t)+\sum_{d \in D n(t)} Q_{d}(t)=\sum_{q \in W Y n(t)} Q_{q}(t) .
$$

Hence, from (5) and (4) it follows (Males et al., 1985; Rossman and Boulos, 1996) that

$$
C_{A, \text { out }, n}(t)=\frac{\sum_{l \in W E n(t)} Q_{l}(t) C_{A, l}(t)+\sum_{d \in D n} Q_{d}(t) C_{A, d}(t)}{\sum_{l \in W E n(t)} Q_{l}(t)+\sum_{d \in D n} Q_{d}(t)} .
$$

The observe that the chorine concentration at the node $n$ at instant $t$ directly depends only on the chlorine concentrations $C_{A, l}(t), l \in W E n(t)$ and $C_{A, d}(t)$, $d \in D n(t)$ at the ends of the pipes supplying water into the node at $t$.

2.2. Quality Model for Estimation Purposes. As was shown in Section 2.1, the DWDS model is composed of the system of algebraic equations that describe the changes in the chlorine concentration at network nodes as well as the system of partial differential equations that describe the distribution of chlorine concentrations along the network pipes. The method of (Mitchel and Griffiths, 1980) can be used in order to discretize (1) in the time and space domains. Here (1) will be discretized only in space and time will remain continuous. The discretization procedure is as follows: Assuming that $z=m \Delta l$, where $\Delta l$ denotes the length of an elementary pipe segment, and standing for $c(t, m)=c(t, m \Delta l)$, Eqn. (1) can be approximated as

$$
\begin{aligned}
& \frac{\mathrm{d} C_{A}(m, t)}{\mathrm{d} t}+v(m, t) \frac{C_{A}(m, t)-C_{A}(m-1, t)}{\Delta l} \\
& -k_{A} C_{A}(m, t)=0 \text {. }
\end{aligned}
$$

Hence (7) describes the chlorine concentration over time at a pipe point located at the distance $m \Delta l$ from the pipe origin. The pipe partitioning into the elementary segments and the models described by (7) are illustrated in Fig. 1.

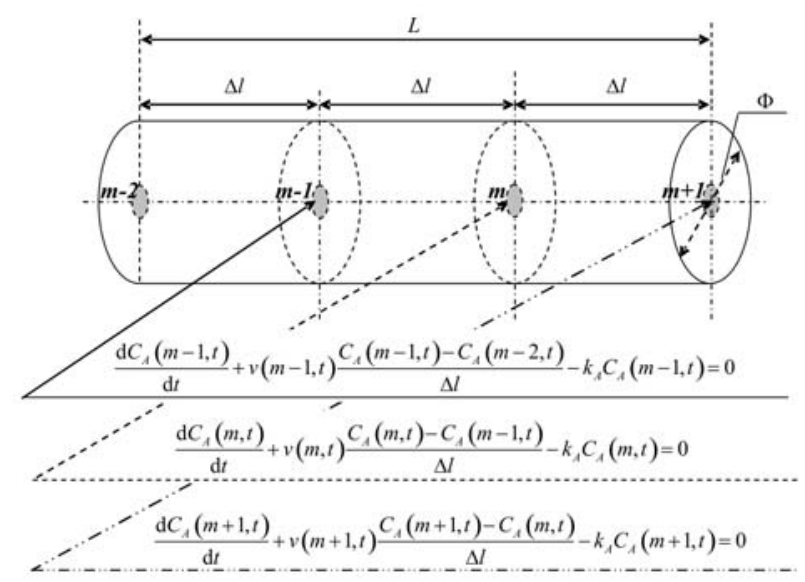

Notation: $L$ - pipe length; $\Phi$ - pipe diameter.

Fig. 1. Example of a pipe quality model.

Note that the linear water flow velocity and flow rate through each segment within a given pipe are the same, i.e., $v(m-1, t)=v(m, t)=v(m+1, t)=v(L, t)$ (see Remark 1). Moreover, the linear water flow velocity and flow rate through a given pipe can be assumed constant over an interval called the hydraulic time step $T_{H}$. The value of $T_{H}$ depends on the demand rate of change and is typically between 0.5 and 2 [h] (Brdys and Ulanicki, 1994). Clearly, the number of constant flow rate intervals over a time horizon $T$ equals $J=T / T_{H}$.

The water quality equation (7) requires determining the length of an elementary pipe segment $\Delta l$ for each pipe. The pipe partitioning method is as follows (Rossman et al., 1993):

Step 1. The travelling time $T_{T}$ is calculated for each pipe over $T_{H}$ :

$$
T_{T, l}=\frac{V_{l}}{Q_{l}},
$$

where $l$ is the pipe number, $V_{l}$ stands for the volume of the $l$-th pipe, $Q_{l}$ denotes the flow rate at the $l$-th pipe over $T_{H}$.

Step 2. A quality step $T_{Q}$ is determined over $T_{H}$ :

$$
T_{Q}=\min _{l} T_{T, l}
$$

Notice that the quality step results from the water quality dynamics and it is assumed that $T_{H}$ is an integer multiple of $T_{Q}$. Typically, in a DWDS, $T_{Q}$ is several minutes. 
Table 1. Data of an exemplary water network.

\begin{tabular}{|c|c|c|c|c|c|c|c|}
\hline \multirow{3}{*}{ Element } & ID & $\begin{array}{c}\text { Elevation } \\
{[\mathrm{m}]}\end{array}$ & $\begin{array}{c}\text { Base } \\
\text { demand } \\
{[\text { LPS }]}\end{array}$ & $\begin{array}{c}\text { Total head } \\
{[\mathrm{m}]}\end{array}$ & $\begin{array}{c}\text { Length } \\
{[\mathrm{m}]}\end{array}$ & $\begin{array}{c}\text { Diameter } \\
{[\mathrm{mm}]}\end{array}$ & Roughness \\
\hline \hline \multirow{6}{*}{ Node } & 2 & 10 & - & - & - & - & - \\
\cline { 2 - 9 } & 3 & 5 & - & - & - & - & - \\
\cline { 2 - 9 } & 4 & 8 & - & - & - & - & - \\
\cline { 2 - 9 } & 5 & 5 & 7 & - & - & - & - \\
\cline { 2 - 9 } & 6 & 5 & 10 & - & - & - & - \\
\hline \multirow{6}{*}{ Peservoipe } & - & - & - & 130 & - & - & - \\
\cline { 2 - 9 } & $1 \mathrm{r}$ & - & - & - & 300 & 100 & 100 \\
\cline { 2 - 8 } & $2 \mathrm{r}$ & - & - & - & 250 & 100 & 100 \\
\cline { 2 - 8 } & $4 \mathrm{r}$ & - & - & - & 150 & 80 & 100 \\
\cline { 2 - 8 } & $5 \mathrm{r}$ & - & - & - & 150 & 80 & 100 \\
\cline { 2 - 8 } & $6 \mathrm{r}$ & - & - & - & 250 & 60 & 100 \\
\cline { 2 - 8 } & $7 \mathrm{r}$ & - & - & - & 250 & 60 & 100 \\
\hline
\end{tabular}

Table 2. Division results for an exemplary network for the first $T_{H}$.

\begin{tabular}{|c|c|c|c|c|}
\hline Pipe ID & $\begin{array}{c}\text { Flow rate } Q \\
{[\text { LPS }]}\end{array}$ & $\begin{array}{c}\text { Travelling time } \\
T_{T}[\mathrm{~min}]\end{array}$ & $\begin{array}{c}\text { Number of } \\
\text { segments } N\end{array}$ & $\begin{array}{c}\text { Segment length } \\
\Delta l[\mathrm{~m}]\end{array}$ \\
\hline \hline $1 \mathrm{r}$ & 5.1 & 7.7 & 2 & 150 \\
\hline $2 \mathrm{r}$ & 2.33 & 14.07 & 3 & 83.33 \\
\hline $3 \mathrm{r}$ & 2.77 & 4.53 & 1 & 150 \\
\hline $4 \mathrm{r}$ & 2.33 & 5.4 & 1 & 150 \\
\hline $5 \mathrm{r}$ & 1.25 & 26.19 & 6 & 41.67 \\
\hline $6 \mathrm{r}$ & 1.52 & 7.73 & 2 & 125 \\
\hline $7 \mathrm{r}$ & 1.48 & 7.98 & 2 & 125 \\
\hline
\end{tabular}

Step 3. The number of segments $N$ for each pipe is calculated over $T_{H}$ :

$$
N_{l}=\operatorname{round}\left(\frac{L_{l} / v_{l}}{T_{Q}}\right),
$$

where $L_{l}$ is the length of the $l$-th pipe, and $v_{l}$ denotes the linear water flow velocity at the $l$-th pipe over $T_{H}$.

Step 4. The length of an elementary pipe segment $\Delta l$ for each pipe is calculated over $T_{H}$ :

$$
\Delta l_{l}=\frac{L_{l}}{N_{l}} .
$$

The above partitioning method is repeated for each $T_{H}$. Hence, a number of segments within a single pipe can be different for each hydraulic step and, therefore, the quality model has a time-varying structure.

Consequently, Eqns. (6) and (7) describe a timecontinuous lumped water quality model for estimation purposes. An example of a DWDS is shown in Fig. 2. The detailed water network data are presented in Table 1. For this water network, the pipes are partitioned as above and the results for the first hydraulic step $T_{H}$ are shown in Table 2.

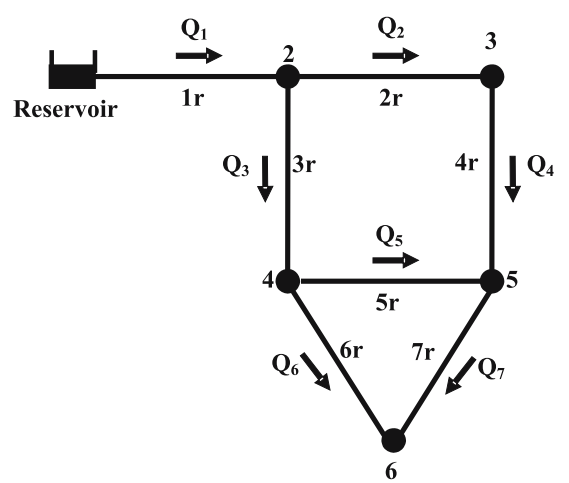

Fig. 2. Exemplary water network. 
The corresponding water quality model was implemented in MATLAB. The model performance was examined by comparing its results with those obtained by applying the well-known EPANET simulator. EPANET accurately integrates the quality model (1) and (6) and the hydraulic model equations that are not presented in the paper in order to determine the flow rates $Q(t)$ and the linear water flow velocities $v(t)$ (Brdys and Ulanicki, 1994). The network quantity-quality model equations can be considered as a faithful representation of reality. Hence, EPANET simulator results will be taken in the paper as the real network responses. In contrast, Eqn. (7) is only an approximation to Eqn. (1). The water quality model described by (6) and (7) is viewed as an approximate model of reality. It is implemented in MATLAB. Hence, in the sequel it is called the MATLAB model. Trajectories of the chlorine concentration at Nodes 3, 5 and 6 are shown in Figs. 3-5, respectively, and a reasonable approximation accuracy of the MATLAB model can be noticed.

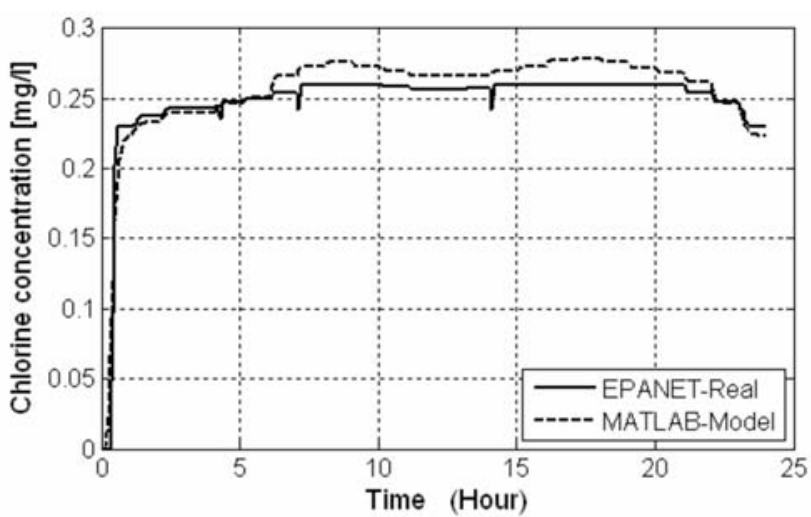

Fig. 3. Trajectory of chlorine concentration at Node 3.

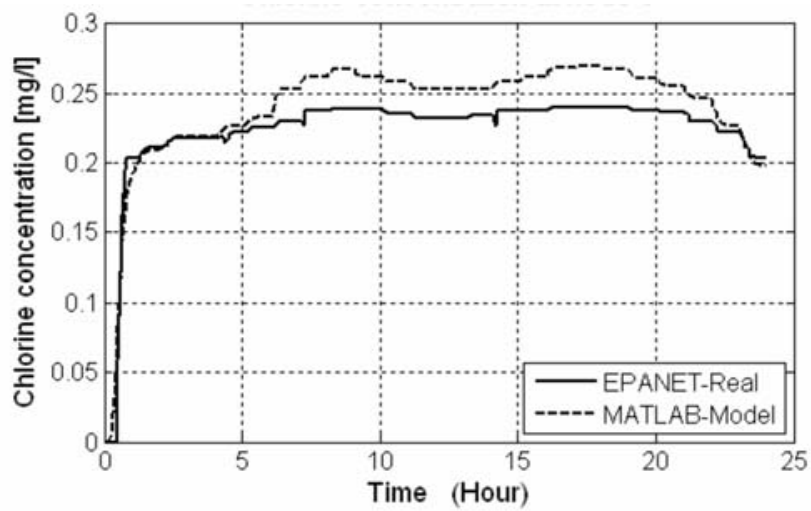

Fig. 4. Trajectory of chlorine concentration at Node 5.

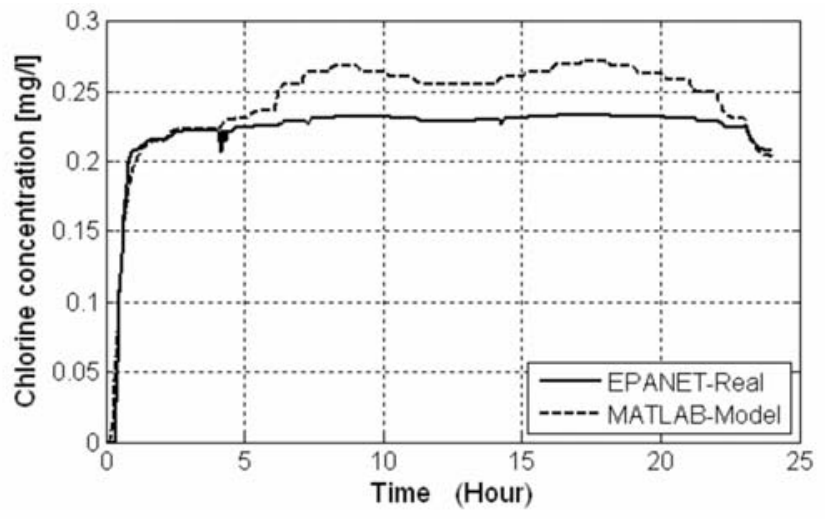

Fig. 5. Trajectory of chlorine concentration at Node 6 .

\section{Examining the Problem of Chlorine Concentration Estimation in DWDS}

The chlorine concentrations at the DWDS nodes are viewed as the system outputs and they can be calculated in accordance with (6). Let $y_{n}(t)$ denote the concentration at the node $n$. Notice that $y_{n}(t)$ can be determined by applying (6). Clearly, the equation structure depends on the node structure. Moreover, the chlorine concentrations at the pipe ends are the quality state variables (see (7)). As presented in Section 2.2, due to different travelling times through the pipes in the DWDS, pipe partitioning into the segments is applied. This introduces new state variables into the quality model. Hence, the quality state $\boldsymbol{x}(t)$ is composed of the chlorine concentrations at all the pipe segment ends and its structure is constant over a hydraulic time step. For the DWDS output, the following holds over the entire horizon:

$$
\boldsymbol{y}(t)=\boldsymbol{C}(t) \boldsymbol{x}(t) .
$$

The state equations can be derived from (7). Denote by $x_{r}(t)$ the chlorine concentration at the end of the pipe segment $r$. From (7), we see that

$$
\frac{\mathrm{d} x_{r}(t)}{\mathrm{d} t}=\left(-\frac{v_{r}(t)}{\Delta l_{r}}+k_{A}\right) x_{r}(t)+\frac{v_{r}(t)}{\Delta l_{r}} C_{i n}(t)
$$

where $v_{r}(t)$ is the linear flow velocity along the segment, $\Delta l_{r}$ denotes the segment length, and $C_{i n}(t)$ signifies the chlorine concentration at the segment beginning.

Three cases for the segment quality input $C_{i n}(t)$ can be distinguished:

(i) $C_{\text {in }}(t)$ equals the chlorine concentration at the end of the segment $r-1$,

(ii) $C_{i n}(t)$ equals the chlorine concentration at the external source, and

(iii) $C_{i n}(t)$ equals the chlorine concentration at the DWDS pipe junction node $p$. 
In Case (i), Eqn. (13) becomes

$$
\frac{\mathrm{d} x_{r}(t)}{\mathrm{d} t}=\left(-\frac{v_{r}(t)}{\Delta l_{r}}+k_{A}\right) x_{r}(t)+\frac{v_{r}(t)}{\Delta l_{r}} x_{r-1}(t)
$$

where $x_{r-1}(t)$ stands for the chlorine concentration at the end of the segment $r-1$.

In Case (ii), Eqn. (13) is

$$
\frac{\mathrm{d} x_{r}(t)}{\mathrm{d} t}=\left(-\frac{v_{r}(t)}{\Delta l_{r}}+k_{A}\right) x_{r}(t)+\frac{v_{r}(t)}{\Delta l_{r}} C_{z r}(t),
$$

where $C_{z r}(t)$ means the chlorine concentration at the external water source.

Finally, in Case (iii), Eqn. (13) becomes

$$
\frac{\mathrm{d} x_{r}(t)}{\mathrm{d} t}=\left(-\frac{v_{r}(t)}{\Delta l_{r}}+k_{A}\right) x_{r}(t)+\frac{v_{r}(t)}{\Delta l_{r}} y_{p}(t),
$$

where $y_{p}(t)$ denotes the chlorine concentration at the pipe junction node $p$.

Finally, on account of (12), the chlorine concentration dynamics throughout the entire DWDS can be written as

$$
\dot{\boldsymbol{x}}(t)=\boldsymbol{A}(t) \boldsymbol{x}(t)+\boldsymbol{b}(t),
$$

where $\boldsymbol{A}(t)$ is the time-varying state matrix, whose elements are composed of hydraulic quantities, lengths of pipes segments and reaction rate coefficients. Furthermore, $\boldsymbol{b}(t)$ is the input vector, whose elements are dependent on the hydraulic quantities, lengths of pipes segments and quality quantities at the reservoirs.

As state variables represent chlorine concentrations at the ends of pipes segments, the chlorine concentrations at certain network nodes only are simultaneously state variables (the state is directly transferred to the system output). This situation occurs at nodes where the water inflow to a node is only from one pipe (Nodes 2, 3 and 4, see Fig. 2). In this case, each of the output measurement equations is as follows:

$$
\boldsymbol{y}(t)=\boldsymbol{x}_{2}(t)
$$

where $\boldsymbol{x}_{2}(t)$ denotes the vector of measurable state variables.

If water is supplied to a node from several pipes, the chlorine concentration at this node is a combination of the state variables. This happens at Nodes 5 and 6.

If all parameters of the model (17) are exactly known, i.e., $\boldsymbol{A}(t)$ and $\boldsymbol{b}(t)$ are exactly known, and the measurement errors are negligible, then a Luenberger observer for the estimation of unmeasured state variables can be used (Luenberger, 1979). In practice, however, most measurements are contaminated by noise and there is also uncertainty in the input vector $\boldsymbol{b}(t)$. In this case, in order to estimate unmeasured state variables, a Kalman filter can be applied. However, the Kalman filter requires specific assumptions regarding the measurement noise and disturbance inputs. In order to satisfy these assumptions, special filters would have to be designed to generate these signals based on the plant input disturbances that are not white, mutually correlated and represented by non-stationary stochastic processes (Grewal and Andrews, 2001). Typically, probabilistic models of uncertainty are used if the assumptions of the Gaussian probability distribution as well as the lack of a time structure of the noise are fulfilled. Unfortunately, in many situations the desired random nature of uncertainty may be questionable. This is because, in reality, the measurement error statistical properties are rarely known and the assumption about the Gaussian probability distribution and the lack of a time structure are rarely valid. This routinely applies to DWDSs (Brdys and Ulanicki, 1994; Chen, 1997). An alternative approach to uncertainty modelling known as the set-membership approach can be found, e.g., (Brdys and Kang, 1994; Milanese et $a l ., 1996)$. In this approach, the uncertainty is described by means of an additive bounded noise where only the bounds are known. The bounds on the measurement errors as well as on the modelling errors are rather easily available, and the bounded models of uncertainty are more realistic because they need less a priori information about the system and they are less demanding than the probabilistic models.

Notice that, in order to solve the water quality model (see Section 2.2), system hydraulic quantities must be available. This kind of information is delivered by a monitoring system of the hydraulics. The monitoring system gathers information from the hydraulic sensors which are used in the DWDS. Typically, only a limited number of hydraulic quantities are directly measured in the DWDS due to high costs of sensors and maintenance. Therefore, in order to overcome the lack of hydraulic information, the necessity of employing a hydraulic estimation based on available hydraulic hard measurements and hydraulic mathematical models arises. A hydraulic estimator based on a set-membership uncertainty modelling can be found in (Brdys and Chen, 1995; Duzinkiewicz, 2006). Hence, the hydraulic estimator delivers estimates of hydraulic quantities in interval form. Clearly, the upper and lower trajectories of hydraulic quantities estimates are constant over the hydraulic steps. It is assumed that hydraulic information in this form is known for the DWDS considered, but it is not obtained from the hydraulic estimator. In order to simulate the estimator, an EPANET DWDS simulator is used. Let assume that $T_{H}=1[\mathrm{~h}]$, $T=24[\mathrm{~h}]$, and for a nominal demand pattern hydraulic simulation results are obtained from EPANET. Next, the values of the hydraulic quantities are distorted by about $\pm \Delta \%$. Hence, the interval form of the hydraulic quantities is as follows:

$$
\begin{aligned}
& Q_{l}^{+}(j)=Q_{l}(j)+\Delta \cdot Q_{l}(j), \\
& Q_{l}^{-}(j)=Q_{l}(j)-\Delta \cdot Q_{l}(j),
\end{aligned}
$$




$$
\begin{aligned}
& v_{l}^{+}(j)=v_{l}(j)+\Delta \cdot v_{l}(j), \\
& v_{l}^{-}(j)=v_{l}(j)-\Delta \cdot v_{l}(j),
\end{aligned}
$$

where $j=1,2,3, \ldots, J, J=T / T_{H}=24[\mathrm{~h}] / 1[\mathrm{~h}]=$ $24, Q_{l}(j)$ is the flow rate gathered from the EPANET simulator for the $l$-th pipe over the $j$-th hydraulic step $T_{H}$, $l=1 r, 2 r, \ldots, 7 r$, and $v_{l}(j)$ is the linear water flow velocity gathered from the EPANET simulator for the $l$-th pipe over the $j$-th hydraulic step $T_{H}, l=1 r, 2 r, \ldots, 7 r$.

In accordance with (19) and (20), the upper and lower trajectories for hydraulic quantities are constant over each hydraulic step $T_{H}$. Nevertheless, over the entire time horizon $T$, these trajectories can be treated as continuous and time varying. It is obvious that the resulting trajectories of hydraulic quantities are more conservative than those that could be obtained by the hydraulic estimator. However, for water quality estimation purposes, the hydraulic information obtained as above is sufficient.

Hence, the uncertainty in (17) is as follows:

(a) The input vector $\boldsymbol{b}(t)$ is not exactly known because the hydraulic quantities and the quality at the reservoir are not exactly known; only lower and upper bounds are available, so that $\boldsymbol{b}^{-}(t) \leq \boldsymbol{b}(t) \leq \boldsymbol{b}^{+}(t)$.

(b) State matrix $\boldsymbol{A}(t)$ is not exactly known, because hydraulic quantities are not exactly known, but certain lower and upper bounds on these quantities are still available; hence $\boldsymbol{A}^{-}(t) \leq \boldsymbol{A}(t) \leq \boldsymbol{A}^{+}(t)$.

Moreover, the state measurement is contaminated by the measurement noise. Hence, (18) is rewritten as

$$
\boldsymbol{y}(t)=\boldsymbol{x}_{2}(t)+\boldsymbol{\varepsilon}(t),
$$

where $\varepsilon(t)$ is the measurement error.

We assume that $\varepsilon(t)$ is bounded, i.e., $|\varepsilon(t)| \leq \varepsilon^{\max }$. Hence, the measured state can be bounded as

$$
\boldsymbol{x}_{2}^{-}(t) \leq \boldsymbol{x}_{2}(t) \leq \boldsymbol{x}_{2}^{+}(t),
$$

where

$$
\boldsymbol{x}_{2}^{-}(t)=\boldsymbol{y}(t)-\boldsymbol{\varepsilon}^{\max }, \quad \boldsymbol{x}_{2}^{+}(t)=\boldsymbol{y}(t)+\boldsymbol{\varepsilon}^{\max } .
$$

Additionally, we assume that, although the state initial conditions are unknown, some bounds are available so that $\boldsymbol{x}^{-}(0) \leq \boldsymbol{x}(0) \leq \boldsymbol{x}^{+}(0)$. Therefore, in order to estimate the chlorine concentration in the DWDS described above, set bounded estimation can be applied. A set bounded estimation algorithm of integrated quantity and quality in a DWDS can be found in (Brdys and Chen, 1996). Unfortunately, this estimation algorithm requires solving on-line highly non-linear and non-convex optimisation tasks. This limits its applicability to on-line monitoring. Therefore, in this paper, an alternative set bounded estimation method that utilizes and further develops an observer known as an interval observer (Gouzé et al., 2000) is proposed.

\section{Interval Observer}

The structures of interval observers can be found in (Gouzé et al., 2000; Alcaraz-González et al., 2004; Rapaport and Dochain, 2005). In (Alcaraz-González et al., 2004), it was shown that, if some conditions are satisfied by the system (24), then the interval observer for this system is stable and robust in spite of the uncertainty in the non-linear part of the system dynamics, inputs and initial conditions. We have

$$
\dot{\boldsymbol{x}}(t)=\boldsymbol{C} \boldsymbol{f}(\boldsymbol{x}(t), t)+\boldsymbol{A}(t) \boldsymbol{x}(t)+\boldsymbol{b}(t),
$$

where $\boldsymbol{x}(t)$ is the state vector, $\boldsymbol{f}(\boldsymbol{x}(t), t)$ denotes an unknown non-linear vector function, $\boldsymbol{C}$ stands for a known matrix, $\boldsymbol{A}(t)$ means the state matrix, and $\boldsymbol{b}(t)$ is the input vector.

The input vector is not exactly known but certain bounds are available so that $\boldsymbol{b}^{-}(t) \leq \boldsymbol{b}(t) \leq \boldsymbol{b}^{+}(t)$. Similarly, the unknown initial conditions are bounded as $\boldsymbol{x}^{-}(0) \leq \boldsymbol{x}(0) \leq \boldsymbol{x}^{+}(0)$. Nevertheless, the linear part of the system dynamics represented by the state matrix $\boldsymbol{A}(t)$ is known and bounded $\boldsymbol{A}^{-} \leq \boldsymbol{A}(t) \leq \boldsymbol{A}^{+}$, and there is no noise in the state measurements. Moreover, in (Rapaport and Dochain, 2005) it was shown that if state measurements are uncertain, the interval observer can still be stable. An application of the interval observer to the wastewater treatment process can be found in (Gouzé et al., 2000; Hadj-Sadok and Gouzé, 2001; Harmand and Rapaport, 2002; Alcaraz-González et al., 2004).

4.1. Interval Observer for a DWDS. For the DWDS presented in Section 2.2, the interval estimator for chlorine monitoring was proposed in (Łangowski and Brdys, 2006):

$$
\begin{aligned}
& \left(\boldsymbol{S}^{+}\right)=\left\{\begin{aligned}
& \dot{\boldsymbol{w}}^{+}(t)=\boldsymbol{A}_{11}^{+}(t) \boldsymbol{w}^{+}(t)+\boldsymbol{A}_{12}^{+}(t) \boldsymbol{x}_{2}^{+}(t) \\
&+\boldsymbol{M} \boldsymbol{v}^{+}(t), \\
& \boldsymbol{w}(0)^{+}= \boldsymbol{N} \boldsymbol{x}(0)^{+} \\
& \hat{\boldsymbol{x}}_{1}^{+}(t)=\boldsymbol{N}_{1}^{-1} \boldsymbol{w}^{+}(t),
\end{aligned}\right. \\
& \left(\boldsymbol{S}^{-}\right)=\left\{\begin{array}{r}
\dot{\boldsymbol{w}}^{-}(t)=\boldsymbol{A}_{11}^{-}(t) \boldsymbol{w}^{-}(t)+\boldsymbol{A}_{12}^{-}(t) \boldsymbol{x}_{2}^{-}(t) \\
+\boldsymbol{M} \boldsymbol{v}^{-}(t) \\
\boldsymbol{w}(0)^{-}=\boldsymbol{N} \boldsymbol{x}(0)^{-}, \\
\hat{\boldsymbol{x}}_{1}^{-}(t)=\boldsymbol{N}_{1}^{-1} \boldsymbol{w}^{-}(t),
\end{array}\right.
\end{aligned}
$$

where $\hat{\boldsymbol{x}}_{1}^{+}(t)$ and $\hat{\boldsymbol{x}}_{1}^{-}(t)$ are respectively upper and lower bounds on the estimated state variables, $\boldsymbol{x}_{2}^{+}(t)$ and $\boldsymbol{x}_{2}^{-}(t)$ are respectively upper and lower bounds on the measured state variables given by (23), $\boldsymbol{w}(t)$ is the auxiliary variable defined as $\boldsymbol{w}(t)=\boldsymbol{N} \boldsymbol{x}(t)$, $\boldsymbol{M}=\left[\begin{array}{l|l|l}\boldsymbol{N}_{1} & \mathbf{0} & \mathbf{0}\end{array}\right], \boldsymbol{N}=\left[\begin{array}{l|l}\boldsymbol{N}_{1} & \mathbf{0}\end{array}\right]$. Moreover, 
$\boldsymbol{N}_{1}=k \boldsymbol{I}$ is a matrix proportional to the identity matrix ( $k$ is an arbitrary positive and constant parameter), and $\boldsymbol{A}_{11}(t), \boldsymbol{A}_{12}(t), \boldsymbol{A}_{21}(t), \boldsymbol{A}_{22}(t)$ are suitable parts of the matrix $\boldsymbol{A}(t)$ structured by the measurement nodes. For example, for one measurement node we have

$$
\boldsymbol{A}(t)=\left[\begin{array}{cccc:c}
\multicolumn{3}{c}{\boldsymbol{A}_{11}(t)} & & \boldsymbol{A}_{12}(t) \\
a_{1,1} & a_{1,2} & \cdots & a_{1, n-1} & a_{1, n} \\
a_{2,1} & a_{2,2} & \cdots & a_{2, n-1} & a_{2, n} \\
\vdots & & \ddots & \vdots & \vdots \\
a_{n-1,1} & a_{n-1,2} & \cdots & a_{n-1, n-1} & a_{n-1, n} \\
\hdashline a_{n, 1} & a_{n, 2} & \cdots & a_{n, n-1} & a_{n, n}
\end{array}\right] .
$$

Furthermore $\boldsymbol{b}_{1}(t), \boldsymbol{b}_{2}(t)$ are suitable parts of the input vector $\boldsymbol{b}(t)$ structured by the measurement nodes. For example, for one measurement node we get

$$
\boldsymbol{b}(t)=\left[\begin{array}{llll:l} 
& & \boldsymbol{b}_{1}(t) & \boldsymbol{b}_{2}(t) \\
b_{1} & b_{2} & \cdots & b_{n-1} & b_{n}
\end{array}\right]^{T},
$$

$$
\begin{aligned}
& \boldsymbol{v}^{+}(t) \\
& =\left[\begin{array}{lll}
\boldsymbol{b}_{1}^{+}(t) & \frac{1}{2}\left(\boldsymbol{b}_{2}^{+}(t)+\boldsymbol{b}_{2}^{-}(t)\right) & \frac{1}{2}\left(\boldsymbol{b}_{2}^{+}(t)-\boldsymbol{b}_{2}^{-}(t)\right)
\end{array}\right]^{T}, \\
& \boldsymbol{v}^{-}(t) \\
& =\left[\begin{array}{lll}
\boldsymbol{b}_{1}^{-}(t) & \frac{1}{2}\left(\boldsymbol{b}_{2}^{+}(t)+\boldsymbol{b}_{2}^{-}(t)\right) & -\frac{1}{2}\left(\boldsymbol{b}_{2}^{+}(t)-\boldsymbol{b}_{2}^{-}(t)\right)
\end{array}\right]^{T} .
\end{aligned}
$$

It is known that the pair of systems $\left(\boldsymbol{S}^{+}, \boldsymbol{S}^{-}\right)$constitutes a truly interval observer generating trajectories $\boldsymbol{x}_{1}^{-}(t)$ and $\boldsymbol{x}_{1}^{+}(t)$ bounding from below and above the estimated state trajectory $\boldsymbol{x}_{1}(t)$, respectively, if each of the systems $\boldsymbol{S}^{+} \boldsymbol{S}^{-}$is cooperative (Gouzé et al. 2000).

Definition 1. (Smith, 1995) The system $\dot{\boldsymbol{\zeta}}=\boldsymbol{f}(\boldsymbol{\zeta}, t)+$ $\boldsymbol{g}(t)$ is said to be cooperative iff its Jacobian matrix satisfies $\partial \boldsymbol{f}_{i}(\boldsymbol{\zeta}, t) / \partial \boldsymbol{\zeta}_{j} \geq 0, \quad \forall i \neq j$.

Notice that the cooperativeness generalizes a monotone property of a system (Smith, 1995). Due to specific properties of the water quality dynamics, the following properties can be shown:

(P1) The hydraulic and quality quantities are nonnegative and bounded. Hence, it is possible to find two constant matrices $\boldsymbol{A}^{-}$and $\boldsymbol{A}^{+}$over the entire time horizon $T$, such that $\boldsymbol{A}^{-} \leq \boldsymbol{A}^{-}(t) \leq \boldsymbol{A}(t) \leq$ $\boldsymbol{A}^{+}(t) \leq \boldsymbol{A}^{+}$.
(P2) The quality model of the above DWDS has cooperative dynamics.

For the water network considered (see Fig. 2), the off-diagonal elements of the Jacobian matrix for the first $T_{H}$ are (see Appendix A):

$$
\begin{gathered}
\frac{v_{l}\left(T_{H}\right)}{\Delta l_{l}\left(T_{H}\right)}, \quad \frac{v_{7 r}\left(T_{H}\right) Q_{4 r}\left(T_{H}\right)}{\Delta l_{7 r}\left(T_{H}\right)\left(Q_{4 r}\left(T_{H}\right)+Q_{5 r}\left(T_{H}\right)\right)}, \\
\frac{v_{7 r}\left(T_{H}\right) Q_{5 r}\left(T_{H}\right)}{\Delta l_{7 r}\left(T_{H}\right)\left(Q_{4 r}\left(T_{H}\right)+Q_{5 r}\left(T_{H}\right)\right)},
\end{gathered}
$$

where $v_{l}\left(T_{H}\right)$ is the linear water flow velocity at the pipe $l[\mathrm{~m} / \mathrm{s}]$ over $T_{H}, l=2 r, \ldots, 6 r, v_{7 r}\left(T_{H}\right)$ denotes the linear water flow velocity at the pipe $7 r[\mathrm{~m} / \mathrm{s}]$ over $T_{H}$, $\Delta l_{l}\left(T_{H}\right)$ means the length of an elementary segment at the pipe $l[\mathrm{~m}]$ over $T_{H}, l=2 r, \ldots, 6 r, \Delta l_{7 r}\left(T_{H}\right)$ signifies the length of an elementary segment at the pipe $7 r$ [m] over $T_{H}$, and $Q_{4 r}\left(T_{H}\right)$, and $Q_{5 r}\left(T_{H}\right)$ are the flow rates at the pipes $4 r$ and $5 r$ [1/s] over $T_{H}$, respectively.

Observe that for successive $T_{H}$, the structures of the off-diagonal elements are the same, but their values may change. This is because the flow rates and linear water flow velocities may change and, consequently, the number and the lengths of pipe segments at each pipe vary in time as well (see Section 2.2). Therefore, the number of state variables may be different over each $T_{H}$. This implies that the dimensions of the state matrix $\boldsymbol{A}(t)$ and the Jacobian matrix may be different over each $T_{H}$. Nevertheless, during estimation over each $T_{H}$ the flow directions remain the same and the above quantities are non-negative and, consequently, the DWDS quality dynamics are cooperative. Moreover, if flow directions change, then, although the quality dynamics model structure change, the values of the off-diagonal elements of the Jacobian matrix remain non-negative.

As Properties P1 and P2 hold, the pair of systems $\left(\boldsymbol{S}^{+}, \boldsymbol{S}^{-}\right)$has cooperative dynamics. Hence, the interval observer (25) produces upper and lower envelopes $\boldsymbol{x}_{1}^{+}(t)$ and $\boldsymbol{x}_{1}^{-}(t)$, respectively, bounding the unmeasured state variables $\boldsymbol{x}_{1}(t)$ in spite of the uncertainty in the inputs (chlorine measurements at reservoirs), initial conditions, state measurements (chlorine measurements at network nodes) and the linear part of the system dynamics (the state matrix $\boldsymbol{A}(t)$ ). A proof that the interval observer (25) is robust and stable is still in progress. However, this postulate is validated by simulation.

The structure of the interval observer (25) requires to determine the matrices $\boldsymbol{A}^{-}(t)$ and $\boldsymbol{A}^{+}(t)$. These matrices are constant over each $T_{H}$. The main parts of these matrices are $\boldsymbol{A}_{11}^{-}(t)$ and $\boldsymbol{A}_{11}^{+}(t)$, respectively. They are presented in Appendix B for the first hydraulic step and sensor localization at Node 3 . Notice that the majority of elements in the matrices $\boldsymbol{A}_{11}^{-}\left(T_{H}\right)$ and $\boldsymbol{A}_{11}^{+}\left(T_{H}\right)$ are directly composed of pipe segments lengths, reaction rate 
coefficients and hydraulic quantities (see Section 3). Due to the flow uncertainties, certain matrix elements are evaluated by solving suitable optimisation problems:

$$
\begin{aligned}
& a_{15,6}^{+}\left(T_{H}\right) \\
& =\frac{1}{\Delta l_{7 r}\left(T_{H}\right)} \max \left(\frac{v_{7 r}\left(T_{H}\right) Q_{4_{r}}\left(T_{H}\right)}{Q_{4 r}\left(T_{H}\right)+Q_{5 r}\left(T_{H}\right)}\right) \\
& \quad=\frac{1}{\Delta l_{7 r}\left(T_{H}\right)} \min \left(\frac{v_{7 r}\left(T_{H}\right) Q_{4 r}\left(T_{H}\right)}{Q_{4 r}\left(T_{H}\right)+Q_{5 r}\left(T_{H}\right)}\right) \\
& a_{15,12}^{+}\left(T_{H}\right) \\
& \quad=\frac{1}{\Delta l_{7 r}\left(T_{H}\right)} \max \left(\frac{v_{7 r}\left(T_{H}\right) Q_{5 r}\left(T_{H}\right)}{Q_{4 r}\left(T_{H}\right)+Q_{5 r}\left(T_{H}\right)}\right) \\
& a_{15,12}^{-}\left(T_{H}\right) \\
& \quad=\frac{1}{\Delta l_{7 r}\left(T_{H}\right)} \min \left(\frac{v_{7 r}\left(T_{H}\right) Q_{5 r}\left(T_{H}\right)}{Q_{4 r}\left(T_{H}\right)+Q_{5 r}\left(T_{H}\right)}\right)
\end{aligned}
$$

where the right-hand sides are minimized and maximized with respect to $v_{7 r}\left(T_{H}\right), Q_{4 r}\left(T_{H}\right)$ and $Q_{5 r}\left(T_{H}\right)$.

Because the upper and lower trajectories of hydraulic quantities are constant over each $T_{H}$, the optimisation problems (27) and (28) are solved only once over each $T_{H}$. Hence, for assumed $T_{H}=1[\mathrm{~h}]$ and $T=24[\mathrm{~h}]$, each of the optimisation problems (27) and (28) is solved 48 times at the $j$-th $\left(j T_{H}\right)$ time instant over $T$. The time index $j$ refers to successive $T_{H}$. Clearly, for $j=1$ we have $t \in[0,1][\mathrm{h}]$, for $j=2$ we have $t \in(1,2][\mathrm{h}], \ldots$, for $j=24$ we have $t \in(23,24]$ [h]. Hence, formulations of the optimisation problems (27) and (28) at each time instant $j \in[1,24]$ are substantiated as follows:

$$
\begin{aligned}
& a_{15,6}^{+}(j) \\
& =\frac{1}{\Delta l_{7 r}(j)} \max _{v_{7 r}(j), Q_{4 r}(j), Q_{5 r}(j)}\left(\frac{v_{7 r}(j) Q_{4 r}(j)}{Q_{4 r}(j)+Q_{5 r}(j)}\right), \\
& a_{15,6}^{-}(j) \\
& =\frac{1}{\Delta l_{7 r}(j)} \min _{v_{7 r}(j), Q_{4 r}(j), Q_{5 r}(j)}\left(\frac{v_{7 r}(j) Q_{4 r}(j)}{Q_{4 r}(j)+Q_{5 r}(j)}\right),
\end{aligned}
$$

subject to

$$
\begin{gathered}
Q_{4 r}^{-}(j) \leq Q_{4 r}(j) \leq Q_{4 r}^{+}(j), \\
Q_{5 r}^{-}(j) \leq Q_{5 r}(j) \leq Q_{5 r}^{+}(j), \\
v_{7 r}^{-}(j) \leq v_{7 r}(j) \leq v_{7 r}^{+}(j),
\end{gathered}
$$

where $j=1,2,3,4, \ldots, 24$.

$$
\begin{aligned}
& a_{15,12}^{+}(j) \\
& \quad=\frac{1}{\Delta l_{7 r}(j)} \max _{v_{r r}(j), Q_{4 r}(j), Q_{5 r}(j)}\left(\frac{v_{7 r}(j) Q_{5 r}(j)}{Q_{4 r}(j)+Q_{5 r}(j)}\right),
\end{aligned}
$$

$$
\begin{aligned}
& a_{15,12}^{-}(j) \\
& \quad=\frac{1}{\Delta l_{7 r}(j)} \min _{v_{7 r}(j), Q_{4 r}(j), Q_{5 r}(j)}\left(\frac{v_{7 r}(j) Q_{5 r}(j)}{Q_{4 r}(j)+Q_{5 r}(j)}\right),
\end{aligned}
$$

subject to

$$
\begin{gathered}
Q_{4 r}^{-}(j) \leq Q_{4 r}(j) \leq Q_{4 r}^{+}(j), \\
Q_{5 r}^{-}(j) \leq Q_{5 r}(j) \leq Q_{5 r}^{+}(j), \\
v_{7 r}^{-}(j) \leq v_{7 r}(j) \leq v_{7 r}^{+}(j),
\end{gathered}
$$

where $j=1,2,3,4, \ldots, 24$.

Note that the matrices $\boldsymbol{A}_{11}^{+}(j)$ and $\boldsymbol{A}_{11}^{-}(j)$ are piecewise constant over $T$.

As was pointed out in Section 3, the quality measurements at the DWDS nodes are at the same time the state measurements only for certain nodes, namely for Nodes 2 , 3 and 4 . Hence, the observer described by (25) is not directly applicable if, e.g., the quality measurements are carried out at Node 5. The proposed interval observer (25) will be modified in Section 4.2 in order to handle the case when a chlorine concentration measurement is carried out at a node with several connected pipes (Node 5).

Clearly, the chlorine concentration is measured only at some time instants. It is assumed that the sampling interval is sufficiently small so that the chlorine concentration over the sampling intervals can be assumed constant. In this paper, the sampling interval is $T_{M Q}=5$ [min]. By the definition of the state variables, the observer (25) returns the quality estimates only at the DWDS nodes that are supplied by one pipe, e.g., at Nodes 2, 3 and 4. The chlorine concentrations at Nodes 5 and 6 are linear combinations of the appropriate state variables. Therefore, the chlorine concentrations at Nodes 5 and 6 are calculated based on the estimates of these state variables by solving the appropriate optimisation problems:

$$
\begin{aligned}
& C_{A, 5}^{-}(t)=\min \left(\frac{Q_{4 r}(t) x_{4 r}(t)+Q_{5 r}(t) x_{5 r}(t)}{Q_{4 r}(t)+Q_{5 r}(t)}\right), \\
& C_{A, 5}^{+}(t)=\max \left(\frac{Q_{4 r}(t) x_{4 r}(t)+Q_{5 r}(t) x_{5 r}(t)}{Q_{4 r}(t)+Q_{5 r}(t)}\right),
\end{aligned}
$$

where the right-hand sides are minimized and maximized with respect to $Q_{4 r}(t), Q_{5 r}(t), x_{4 r}(t), x_{5 r}(t)$.

$$
\begin{aligned}
& C_{A, 6}^{-}(t)=\min \left(\frac{Q_{6 r}(t) x_{6 r}(t)+Q_{7 r}(t) x_{7 r}(t)}{Q_{6 r}(t)+Q_{7 r}(t)}\right), \\
& C_{A, 6}^{+}(t)=\max \left(\frac{Q_{6 r}(t) x_{6 r}(t)+Q_{7 r}(t) x_{7 r}(t)}{Q_{6 r}(t)+Q_{7 r}(t)}\right),
\end{aligned}
$$

where the right-hand sides are minimized and maximized with respect to $Q_{6 r}(t), Q_{7 r}(t), x_{6 r}(t), x_{7 r}(t)$.

The optimisation problems (31) and (32) are different from the optimisation problems (27) and (28) because, 
apart from the hydraulic quantities, they also involve estimates of the state variables $x_{4 r}(t), x_{5 r}(t), x_{6 r}(t), x_{7 r}(t)$ representing chlorine concentrations at the ends of pipes: $4 r, 5 r, 6 r$ and $7 r$, respectively. The optimisation tasks are solved at discrete time instants imposed by the quality sampling interval to produce the estimates at these time instants. Hence, for assumed $T_{M Q}=5[\mathrm{~min}], T_{H}=1[\mathrm{~h}]$ and $T=24[\mathrm{~h}]$, the optimisation problems (31) and (32) are solved 289 times. Clearly, for $k=0$ we have $t=$ 0 [min], for $k=1$ we have $t=5[\min ], \ldots$, for $k=288$ we have $t=1440$ [min]. Hence, the formulation of the optimisation problems (31) and (32) over $T$ is as follows:

$$
\begin{aligned}
C_{A, 5}^{+}(k)= & \max _{Q_{4 r}(j), Q_{5 r}(j), x_{4 r}(k), x_{5 r}(k)} \\
& \left(\frac{Q_{4 r}(j) x_{4 r}(k)+Q_{5 r}(j) x_{5 r}(k)}{Q_{4 r}(j)+Q_{5 r}(j)}\right), \\
C_{A, 5}^{-}(k)= & \min _{Q_{4 r}(j), Q_{5 r}(j), x_{4 r}(k), x_{5 r}(k)} \\
& \left(\frac{Q_{4 r}(j) x_{4 r}(k)+Q_{5 r}(j) x_{5 r}(k)}{Q_{4 r}(j)+Q_{5 r}(j)}\right),
\end{aligned}
$$

subject to

$$
\begin{aligned}
& Q_{4 r}^{-}(j) \leq Q_{4 r}(j) \leq Q_{4 r}^{+}(j), \\
& Q_{5 r}^{-}(j) \leq Q_{5 r}(j) \leq Q_{5 r}^{+}(j), \\
& x_{4 r}^{-}(k) \leq x_{4 r}(k) \leq x_{4 r}^{+}(k), \\
& x_{5 r}^{-}(k) \leq x_{5 r}(k) \leq x_{5 r}^{+}(k),
\end{aligned}
$$

with respect to

$$
j= \begin{cases}1 & \text { if } k \geq 0 \text { and } k \leq 12, \\ j+1 & \text { if } k>12 \text { and } \bmod (k, 12)=1\end{cases}
$$

where $k=0,1, \ldots, 288$, and $j=1,2, \ldots, 24$,

$$
\begin{aligned}
C_{A, 6}^{+}(k)= & \max _{Q_{6 r}(j), Q_{7 r}(j), x_{6 r}(k), x_{7 r}(k)} \\
& \left(\frac{Q_{6 r}(j) x_{6 r}(k)+Q_{7 r}(j) x_{7 r}(k)}{Q_{6 r}(j)+Q_{7 r}(j)}\right), \\
C_{A, 6}^{-}(k)= & \min _{Q_{6 r}(j), Q_{7 r}(j), x_{6 r}(k), x_{7 r}(k)} \\
& \left(\frac{Q_{6 r}(j) x_{6 r}(k)+Q_{7 r}(j) x_{7 r}(k)}{Q_{6 r}(j)+Q_{7 r}(j)}\right),
\end{aligned}
$$

subject to

$$
\begin{aligned}
& Q_{6 r}^{-}(j) \leq Q_{6 r}(j) \leq Q_{6 r}^{+}(j), \\
& Q_{7 r}^{-}(j) \leq Q_{7 r}(j) \leq Q_{7 r}^{+}(j), \\
& x_{6 r}^{-}(k) \leq x_{6 r}(k) \leq x_{6 r}^{+}(k), \\
& x_{7 r}^{-}(k) \leq x_{7 r}(k) \leq x_{7 r}^{+}(k),
\end{aligned}
$$

with respect to

$$
j= \begin{cases}1 & \text { if } k \geq 0 \text { and } k \leq 12 \\ j+1 & \text { if } k>12 \text { and } \bmod (k, 12)=1\end{cases}
$$

where $k=0,1, \ldots, 288$, and $j=1,2, \ldots, 24$.

Clearly, the above is time consuming and the following can be tried instead:

$$
\begin{aligned}
& C_{A, 5}^{-}(t)=\frac{Q_{4 r}^{-}(t) x_{4 r}^{-}(t)+Q_{5 r}^{-}(t) x_{5 r}^{-}(t)}{Q_{4 r}^{+}(t)+Q_{5 r}^{+}(t)}, \\
& C_{A, 5}^{+}(t)=\frac{Q_{4 r}^{+}(t) x_{4 r}^{+}(t)+Q_{5 r}^{+}(t) x_{5 r}^{+}(t)}{Q_{4 r}^{-}(t)+Q_{5 r}^{-}(t)}, \\
& C_{A, 6}^{-}(t)=\frac{Q_{6 r}^{-}(t) x_{6 r}^{-}(t)+Q_{7 r}^{-}(t) x_{7 r}^{-}(t)}{Q_{6 r}^{+}(t)+Q_{7 r}^{+}(t)}, \\
& C_{A, 6}^{+}(t)=\frac{Q_{6 r}^{+}(t) x_{6 r}^{+}(t)+Q_{7 r}^{+}(t) x_{7 r}^{+}(t)}{Q_{6 r}^{-}(t)+Q_{7 r}^{-}(t)} .
\end{aligned}
$$

In a much the same way, the optimisation problems (27) and (28) can be rewritten as

$$
\begin{aligned}
a_{15,6}^{+}\left(T_{H}\right) & =\frac{1}{\Delta l_{7 r}\left(T_{H}\right)}\left(\frac{v_{7 r}^{+}\left(T_{H}\right) Q_{4 r}^{+}\left(T_{H}\right)}{Q_{4 r}^{-}\left(T_{H}\right)+Q_{5 r}^{-}\left(T_{H}\right)}\right), \\
a_{15,6}^{-}\left(T_{H}\right) & =\frac{1}{\Delta l_{7 r}\left(T_{H}\right)}\left(\frac{v_{7 r}^{-}\left(T_{H}\right) Q_{4 r}^{-}\left(T_{H}\right)}{Q_{4 r}^{+}\left(T_{H}\right)+Q_{5 r}^{+}\left(T_{H}\right)}\right),(37) \\
a_{15,12}^{+}\left(T_{H}\right) & =\frac{1}{\Delta l_{7 r}\left(T_{H}\right)}\left(\frac{v_{7 r}^{+}\left(T_{H}\right) Q_{5 r}^{+}\left(T_{H}\right)}{Q_{4 r}^{-}\left(T_{H}\right)+Q_{5 r}^{-}\left(T_{H}\right)}\right), \\
a_{15,12}^{-}\left(T_{H}\right) & =\frac{1}{\Delta l_{7 r}\left(T_{H}\right)}\left(\frac{v_{7 r}^{-}\left(T_{H}\right) Q_{5 r}^{-}\left(T_{H}\right)}{Q_{4 r}^{+}\left(T_{H}\right)+Q_{5 r}^{+}\left(T_{H}\right)}\right) .
\end{aligned}
$$

Clearly, using the relationships (35)-(38) introduces a conservatism into the estimates that can be significant.

4.2. Interval Observer for a DWDS with Quality Measurements at a Node Supplied by Several Pipes. Let us consider the case where a chlorine concentration sensor is located at Node 5 (see Fig. 2). The chlorine concentration at Node 5 is described by (cf. Section 2.1):

$$
C_{A, 5}(t)=\frac{Q_{4 r}(t) x_{4 r}(t)+Q_{5 r}(t) x_{5 r}(t)}{Q_{4 r}(t)+Q_{5 r}(t)} .
$$

The measurement of $C_{A, 5}(t)$ cannot be directly used by the observer as it is not a state variable measurement. However, by (39), the state variable $x_{5 r}(t)$ can be expressed as

$$
x_{5 r}(t)=\frac{Q_{4 r}(t)}{Q_{5 r}(t)} C_{A, 5}(t)+C_{A, 5}(t)-\frac{Q_{4 r}(t)}{Q_{5 r}(t)} x_{4 r}(t)
$$

to define a pseudomeasurement $\tilde{x}_{5 r}(t)$ indirectly measured $x_{5 r}(t)$ as

$$
\tilde{x}_{5 r}(t)=\frac{Q_{4 r}(t)}{Q_{5 r}(t)} C_{A, 5}(t)+C_{A, 5}(t)-\frac{Q_{4 r}(t)}{Q_{5 r}(t)} \hat{x}_{4 r}(t) .
$$


Table 3. Solver efficiency for an examplary network.

\begin{tabular}{|l|c|c|c|c|c|c|c|c|}
\hline & \multicolumn{2}{|c|}{$\begin{array}{l}\text { Optimisation } \\
\text { problem (16) }\end{array}$} & \multicolumn{2}{c|}{$\begin{array}{l}\text { Optimisation } \\
\text { problem (17) }\end{array}$} & \multicolumn{2}{c|}{$\begin{array}{l}\text { Optimisation } \\
\text { problem (22) }\end{array}$} & \multicolumn{2}{c|}{$\begin{array}{l}\text { Optimisation } \\
\text { problem (23) }\end{array}$} \\
\cline { 2 - 11 } & $\min$ & $\max$ & $\min$ & $\max$ & $\min$ & $\max$ & $\min$ & $\max$ \\
\hline $\begin{array}{l}\text { Number of } \\
\text { decision variables }\end{array}$ & 3 & 3 & 3 & 3 & 4 & 4 & 4 & 4 \\
\hline Number of constraints & 6 & 6 & 6 & 6 & 8 & 8 & 8 & 8 \\
\hline $\begin{array}{l}\text { Number of single } \\
\text { optimizations (over 24 [h]) }\end{array}$ & 24 & 24 & 24 & 24 & 289 & 289 & 289 & 289 \\
\hline $\begin{array}{l}\text { Average computation time } \\
\text { for single optimization } \\
\text { [sec] } \times 10^{-3}\end{array}$ & 8 & 8 & 8 & 8 & 9 & 9 & 9 & 9 \\
\hline
\end{tabular}

The needed upper and lower bounds on $\tilde{x}_{5 r}(t)$ are calculated from (41) as follows:

$$
\begin{aligned}
& \tilde{x}_{5 r}^{+}(t)=C_{A, 5}^{+}(t) \frac{Q_{4 r}^{+}(t)}{Q_{5 r}^{-}(t)}+C_{A, 5}^{+}(t)-\hat{x}_{4 r}^{-}(t) \frac{Q_{4 r}^{-}(t)}{Q_{5 r}^{+}(t)} \\
& \tilde{x}_{5 r}^{-}(t)=C_{A, 5}^{-}(t) \frac{Q_{4 r}^{-}(t)}{Q_{5 r}^{+}(t)}+C_{A, 5}^{-}(t)-\hat{x}_{4 r}^{+}(t) \frac{Q_{4 r}^{+}(t)}{Q_{5 r}^{-}(t)}
\end{aligned}
$$

The state variable $x_{5 r}(t)$ is now removed from the estimated states, and the vector of hard measurements $\boldsymbol{x}_{2}(t)$ is augmented by adding the pseudomeasurement $\tilde{x}_{5 r}(t)$ to produce the interval observer according to (25). The analysis of the observer properties including stability is under way. Note that there is now a feedback from the state estimates into the measurements that requires attention from the stability point of view.

\section{Efficiency of the Interval Estimation Algorithm}

It is obvious that for on-line estimation purposes the efficiency of the estimation algorithm should be high. The computation time of the estimated chlorine concentration bounds for each hydraulic step $T_{H}$ is about 3 [sec]. Note that during the estimation process the optimisation problems (29) and (30) as well as (33) and (34) are solved. The following properties characterize the optimisation problems (29) and (30), which are solved in the $j$-th step, as well as the optimisation problems (33) and (34), which are solved in the $k$-th step:

- non-linear objective function,

- inequality linear constrains,

- continuous decision variables.

In this paper, in order to solve these optimisation problems, the SQP (Sequential Quadratic Programming) solver is used. The SQP solver is implemented in the MATLAB optimisation toolbox. This solver is called by the MATLAB function fmincon. The average computation times (in seconds) of solving a single optimisation task are shown in Table 3.
The above refers to the following settings:

- simulation environment: Matlab/Simulink V. 7.1 (R14) Service Pack 3,

- operating system: Microsoft Windows XP Professional Service Pack 2,

- hardware set-up: Intel Pentium 4, 3.00 GHz CPU, 1.00 GB RAM.

Clearly, the average computation time for a single optimisation task will increase for larger networks due to an increased number of nodes. The computation time associated with a single node will remain the same, i.e., about 0.009 [sec], as long as there are up to two pipes supplying water. It will increase, but not significantly, if the number of such pipes increases.

\section{Simulation Results}

This section presents simulation results for an exemplary DWDS illustrated in Fig. 2. It is composed of one water source, seven pipes and five nodes. The flow directions remain the same during the network operation and they are clearly marked in Fig. 2. Nodes 5 and 6 are the water demand nodes. The nominal and real demand patterns at these nodes are the same and are shown in Fig. 6. Both demand patterns represent typical demand loads: two peaks of water consumption can be seen. The nominal demand pattern is used in order to obtain the nominal values of hydraulic quantities and then they are distorted by about $\pm \Delta \%$ (see Section 3 ). The chlorine concentration measurements and chlorine concentration at the reservoir are provided by a real EPANET simulator. Clearly, EPANET with real demand pattern scenarios is used in order to generate the chlorine concentration measurements. The measurements are contaminated by measurement noise (see (21)).

6.1. Estimation Results at Unmeasured Nodes. In this section, an application of the interval observer for estimating the chlorine concentration is presented. The chlorine concentration sensor is placed at Node 3 . The es- 


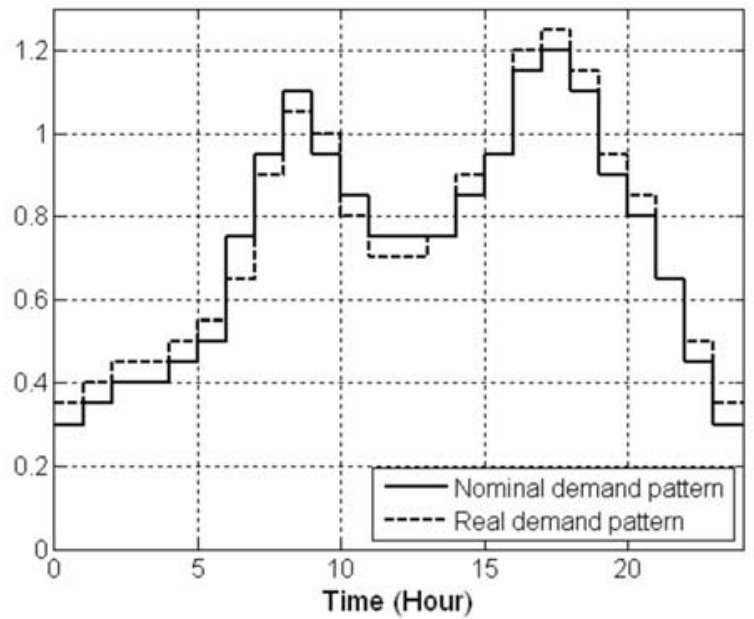

Fig. 6. Demand pattern at Nodes 5 and 6.

timation results at Nodes 5 and 6 are illustrated in Figs. 7 to 14 , and they are compared with the real EPANET results. Four trajectories are presented in each figure: the chlorine concentration from real EPANET, bounds on the estimated chlorine concentration and the Chebyshev centres. Uncertainties in the quantity and quality parameters have significant impact on the estimation performance. For the estimation carried out at Nodes 5 and 6, this is illustrated in Figs. 7 to 10 and Figs. 11 to 14, respectively. The uncertainty is introduced by distorting the water quality and quantity by about $\pm 5 \%$ and $\pm 10 \%$. The initial conditions for the calculation of the upper and lower chlorine concentration bounds are $0.1[\mathrm{mg} / \mathrm{l}]$ and 0 [mg/l], respectively.

The real chlorine concentration trajectory is always inside the estimation bounds. The uncertainty influences the bound radius as expected. That is, if uncertainty increases, then the estimation bounds are widened. Note that the magnitude of uncertainty in hydraulic quantities has crucial impact on estimation performance.

If (35)-(38) are used instead of the optimisation

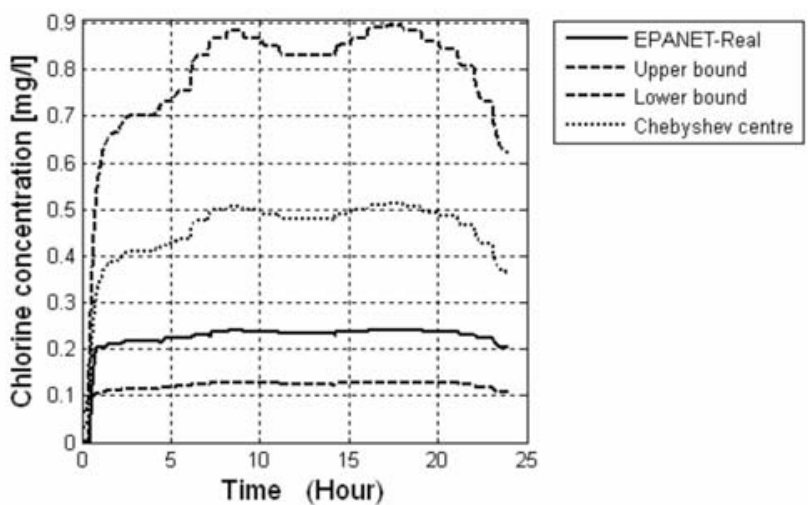

Fig. 7. Interval observer estimation results at Node 5 under $\pm 10 \%$ uncertainty in hydraulic and quality quantities.

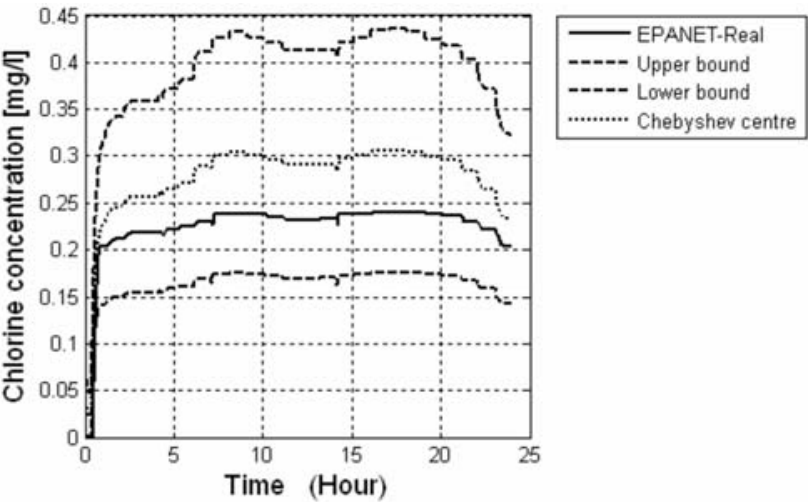

Fig. 8. Interval observer estimation results at Node 5 under $\pm 5 \%$ uncertainty in hydraulic and quality quantities.

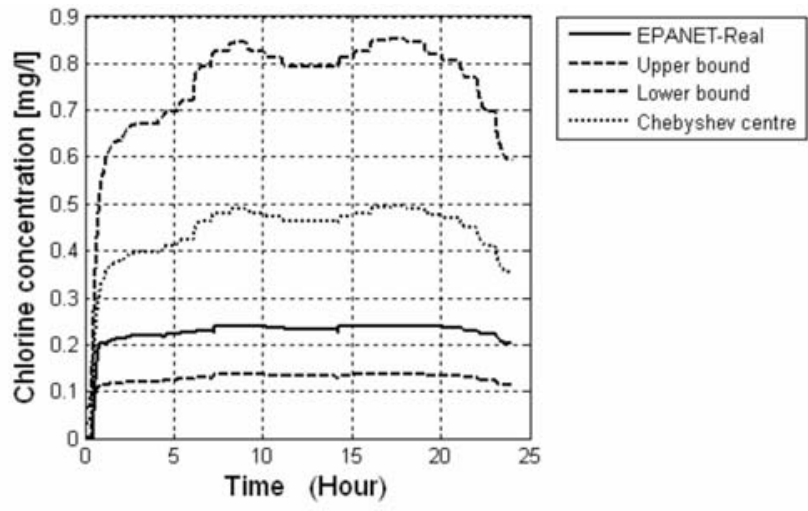

Fig. 9. Interval observer estimation results at Node 5 under $\pm 10 \%$ uncertainty in hydraulic quantities and $\pm 5 \%$ uncertainty in quality quantities.

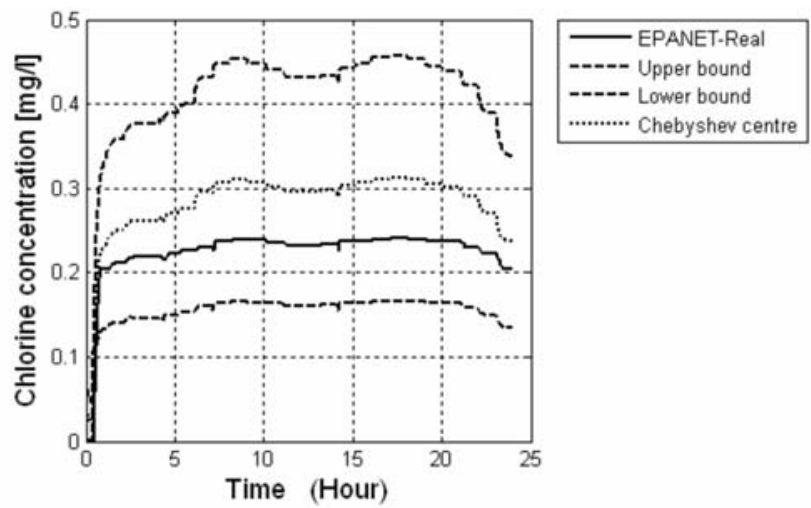

Fig. 10. Interval observer estimation results at Node 5 under $\pm 5 \%$ uncertainty in hydraulic quantities and $\pm 10 \%$ uncertainty in quality quantities.

problems (29)-(30) and (33)-(34), the estimation bounds are widened. For this case, the estimation results at Nodes 5 and 6 are shown in Figs. 15 and 16, respectively. Similarly, four trajectories are presented in each figure: the chlorine concentration from real EPANET, bounds on the estimated chlorine concentration and the Chebyshev 

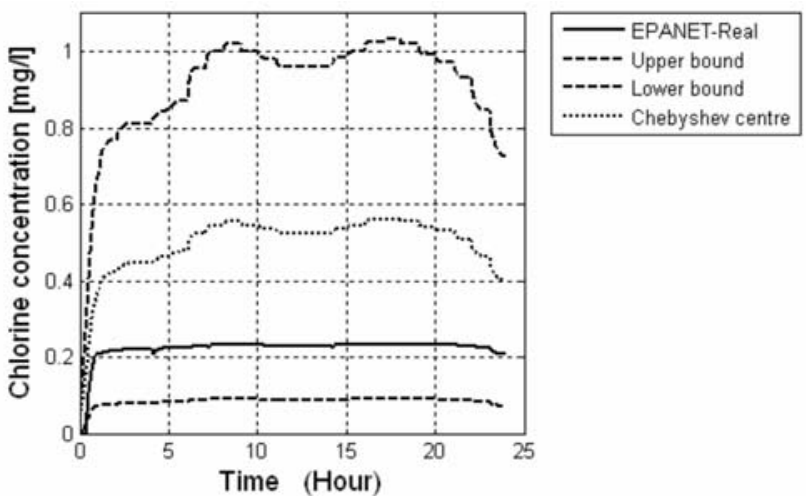

Fig. 11. Interval observer estimation results at Node 6 under $\pm 10 \%$ uncertainty in hydraulic and quality quantities.

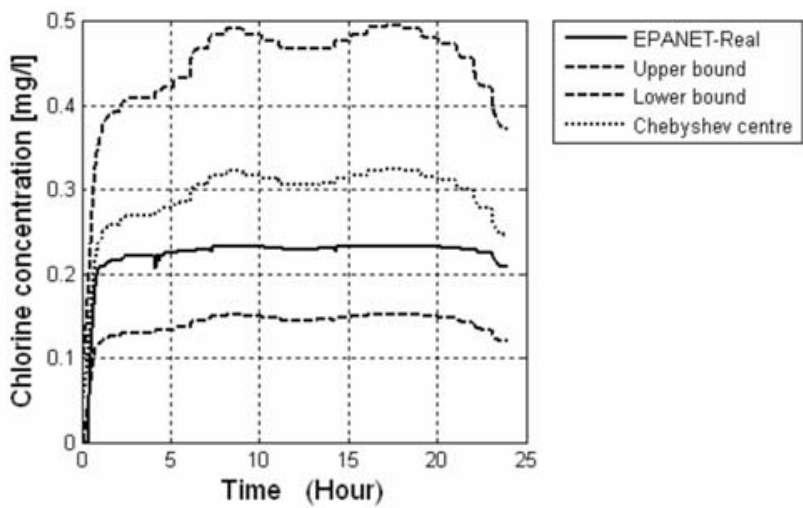

Fig. 12. Interval observer estimation results at Node 6 under $\pm 5 \%$ uncertainty in hydraulic and quality quantities.

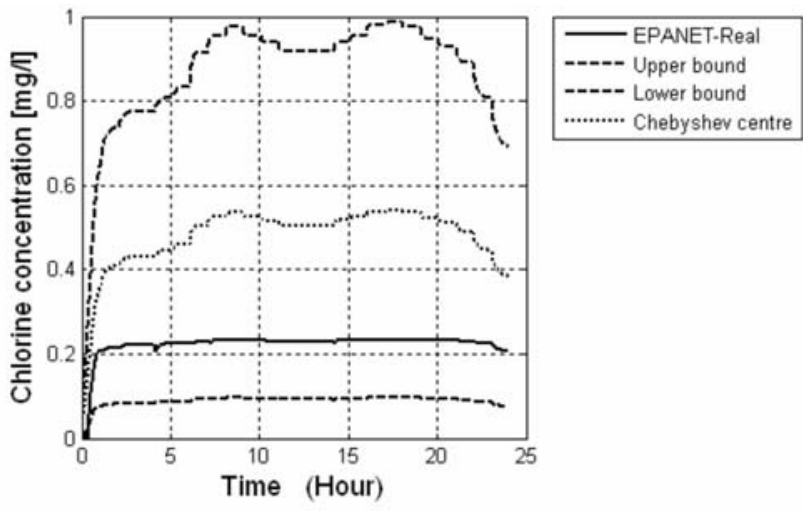

Fig. 13. Interval observer estimation results at Node 6 under $\pm 10 \%$ uncertainty in hydraulic quantities and $\pm 5 \%$ uncertainty in quality quantities.

centres. The $\pm 5 \%$ uncertainty is in hydraulic and quality quantities.

A comparison of the estimated chlorine concentration bounds at Node 5 with and without solving the optimisation problems is illustrated in Fig. 17. The worse estimation performance without solving the optimisation problems can be noticed in Fig. 17. In this case, the only advantage during the estimation process is reduction in computation time.

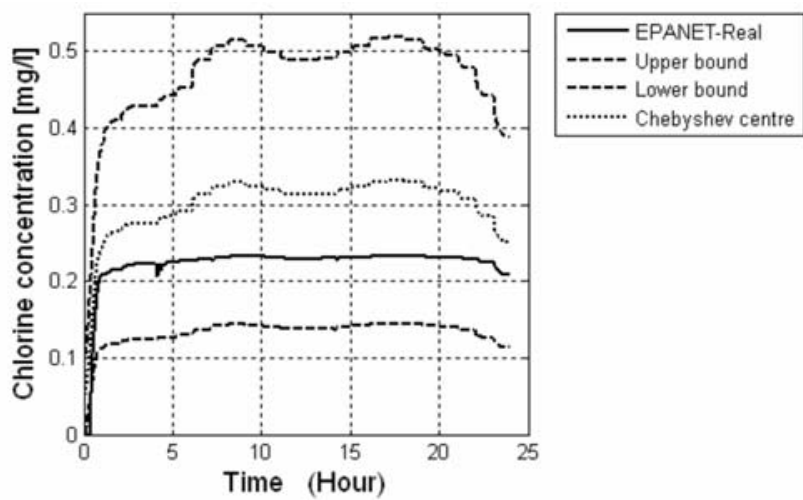

Fig. 14. Interval observer estimation results at Node 6 under $\pm 5 \%$ uncertainty in hydraulic quantities and $\pm 10 \%$ uncertainty in quality quantities.

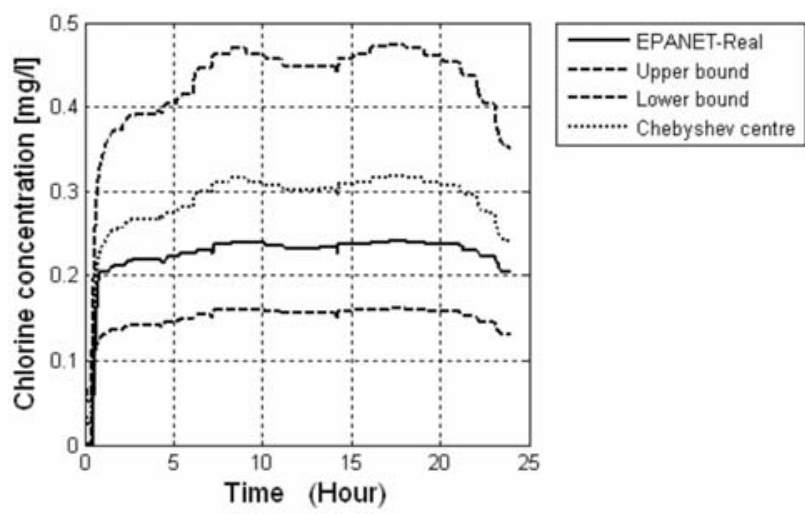

Fig. 15. Interval observer estimation results at Node 5 without solving the optimisation problems under $\pm 5 \%$ uncertainty in hydraulic and quality quantities.

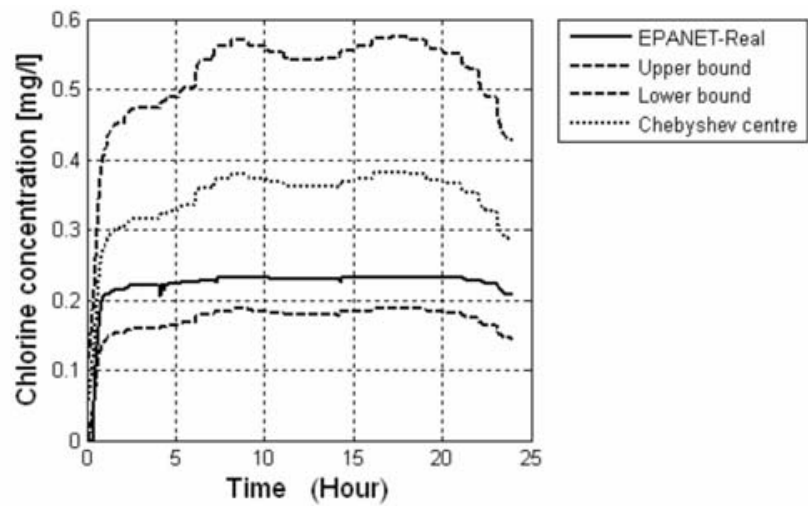

Fig. 16. Interval observer estimation results at Node 6 without solving the optimisation problems under $\pm 5 \%$ uncertainty in hydraulic and quality quantities.

6.2. Estimation Results at Measurement Nodes. In the presented set bounded estimation method, the estimation of the chlorine concentration at a measurement node (Node 3) is not performed directly. Nevertheless, once the quality estimates at the unmeasured nodes have been produced by the observer, upper and lower bounding tra- 


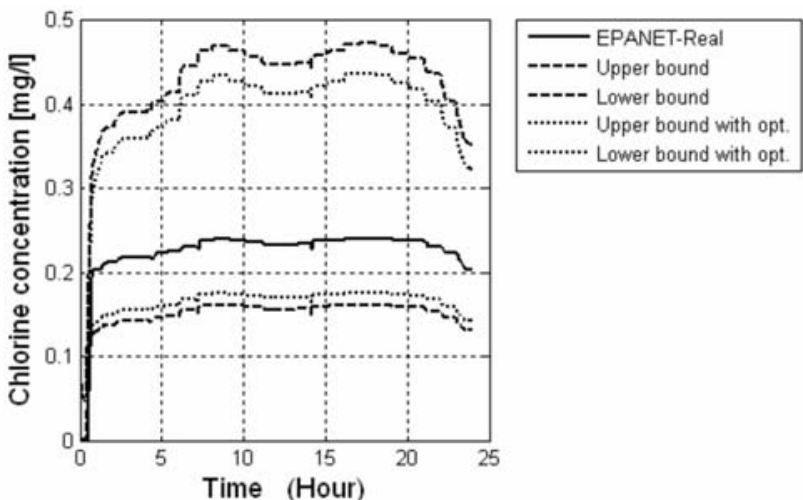

Fig. 17. Comparison of estimation performance at Node 5 under $\pm 5 \%$ uncertainty in hydraulic and quality quantities.

jectories on the chlorine concentration at the measurement node can be calculated by solving the following two differential equations:

$$
\begin{aligned}
& \dot{\hat{x}}_{2 r}^{+}(t)=\left(-\frac{v_{2 r}^{-}(t)}{\Delta l_{2 r}(t)}+k_{A}\right) \hat{x}_{2 r}^{+}(t)+\frac{v_{2 r}^{+}(t)}{\Delta l_{2 r}(t)} \hat{x}_{2 r-1}^{+}(t), \\
& \dot{\hat{x}}_{2 r}^{-}(t)=\left(-\frac{v_{2 r}^{+}(t)}{\Delta l_{2 r}(t)}+k_{A}\right) \hat{x}_{2 r}^{-}(t)+\frac{v_{2 r}^{-}(t)}{\Delta l_{2 r}(t)} \hat{x}_{2 r-1}^{-}(t),
\end{aligned}
$$

where $\hat{x}_{2 r}^{+}(t)$ and $\hat{x}_{2 r}^{-}(t)$ are respectively upper and lower trajectories bounding the chlorine concentration at the end of the last segment of the pipe $2 r$, and $\hat{x}_{2 r-1}^{+}(t)$ and $\hat{x}_{2 r-1}^{-}(t)$ are respectively upper and lower trajectories bounding the chlorine concentration at the end of the segment of the pipe $2 r$, which is adjacent to the last segment.

Estimation results at Node 3 under $\pm 5 \%$ uncertainty in hydraulic quantities and quality quantities are shown in Fig. 18. Five trajectories are illustrated in Fig. 18: upper and lower bounds on the real chlorine concentration measurements (from real EPANET) at Node 3, bounds on the estimated chlorine concentration at Node 3 and the Chebyshev centre.

Note that the measurement bounds on $C_{A, 3}(t)$ are much tighter than the post-estimated ones produced by (43) and (44). However, this may not be so in general. Hence, a sensible approach is to take an intersection of the two bounds to produce the final estimates at the measurement nodes.

6.3. Number and Location of Sensors. In this section, the performance of the proposed set bounded estimation method is examined with respect to the number and location of sensors. All the estimation results presented in this section are under $\pm 5 \%$ uncertainty in hydraulic and quality quantities. The estimation results at Nodes 5 and 6

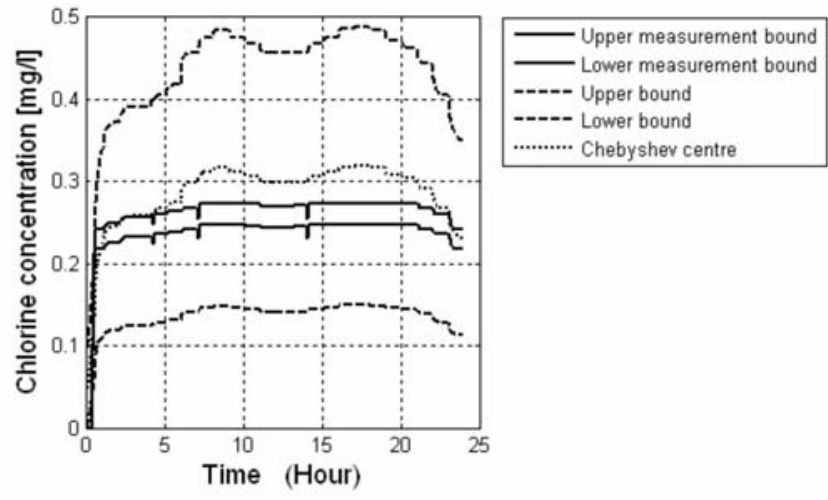

Fig. 18. Interval observer estimation results at Node 3 under $\pm 5 \%$ uncertainty in hydraulic and quality quantities.

when a chlorine concentration sensor is located at Node 3 are shown in Figs. 8 and 12, respectively. A similar situation is presented in Figs. 19 and 20, respectively, while the sensor is located at Node 4 instead of Node 3. Finally, the estimation results at Nodes 5 and 6 for the sensor placement at Nodes 3 and 4 are shown in Figs. 21 and 22, respectively.

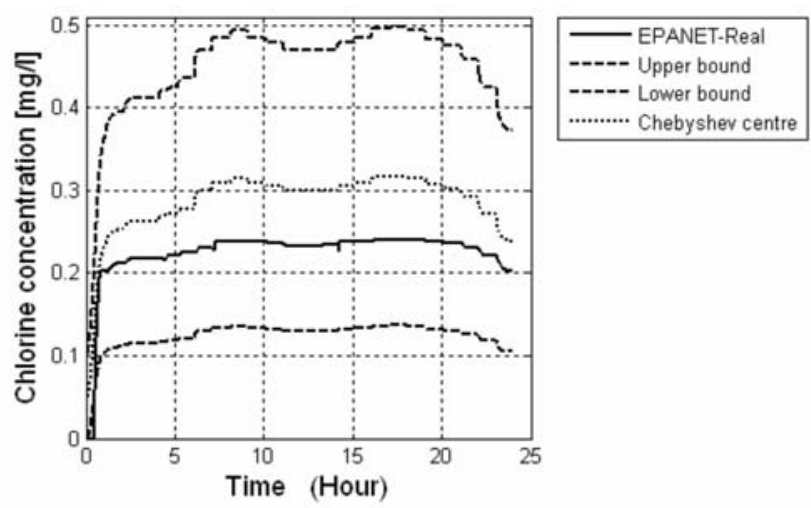

Fig. 19. Interval observer estimation results at Node 5 under $\pm 5 \%$ uncertainty in hydraulic and quality quantities (the measurement node is Node 4).

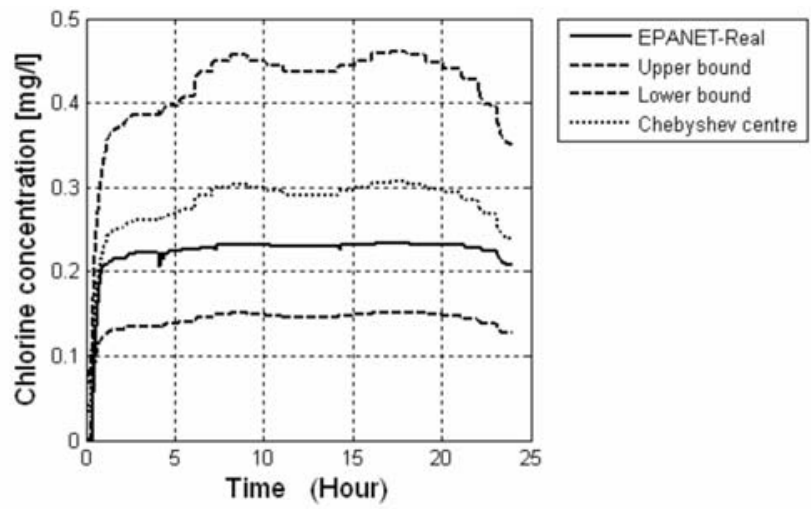

Fig. 20. Interval observer estimation results at Node 6 under $\pm 5 \%$ uncertainty in hydraulic and quality quantities (the measurement node is Node 4). 


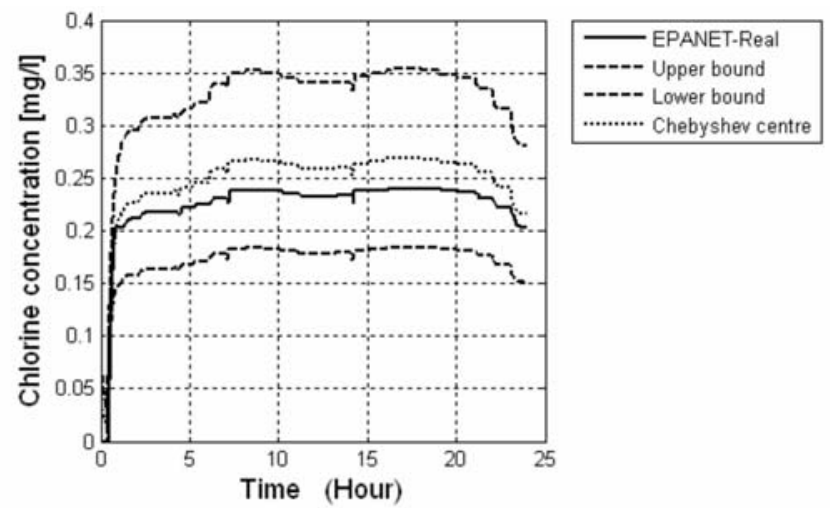

Fig. 21. Interval observer estimation results at Node 5 under $\pm 5 \%$ uncertainty in hydraulic and quality quantities (the measurement nodes are Nodes 3 and 4).
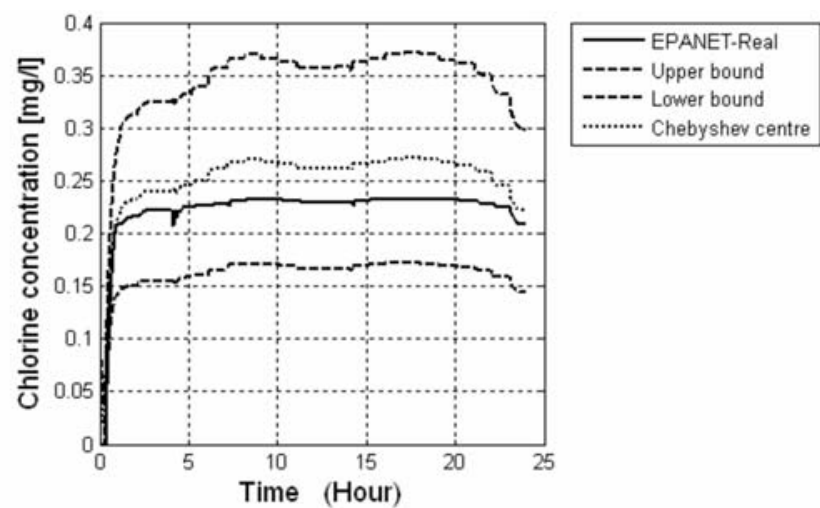

Fig. 22. Interval observer estimation results at Node 6 under $\pm 5 \%$ uncertainty in hydraulic and quality quantities (the measurement nodes are Nodes 3 and 4).

A comparison of estimation performance at Nodes 5 and 6 for various locations and numbers of sensors is presented in Figs. 23 and 24, respectively.

The simulation results presented above show that the location and number of sensors have crucial impact on estimation performance. Notice that real conditions in the DWDS may be considerably different from nominal ones. Clearly, real demand patterns at consumer nodes may differ from nominal demand patterns more than it is presented in Fig. 6. Moreover, the reaction rate coefficients may change, etc. In these cases, the presented water quality estimator is better than the water quality simulator (the open-loop estimator). This is because during the estimation process, in addition to the quality model, also current chlorine concentration measurements are utilized. This is illustrated in Figs. 25 and 26. The trajectories of the real chlorine concentration, the nominal chlorine concentration (the open-loop estimator) and the Chebyshev centre at Nodes 5 and 6 are shown in Figs. 25 and 26, respectively. The chlorine concentration sensors are placed at Nodes 3 and 4 .

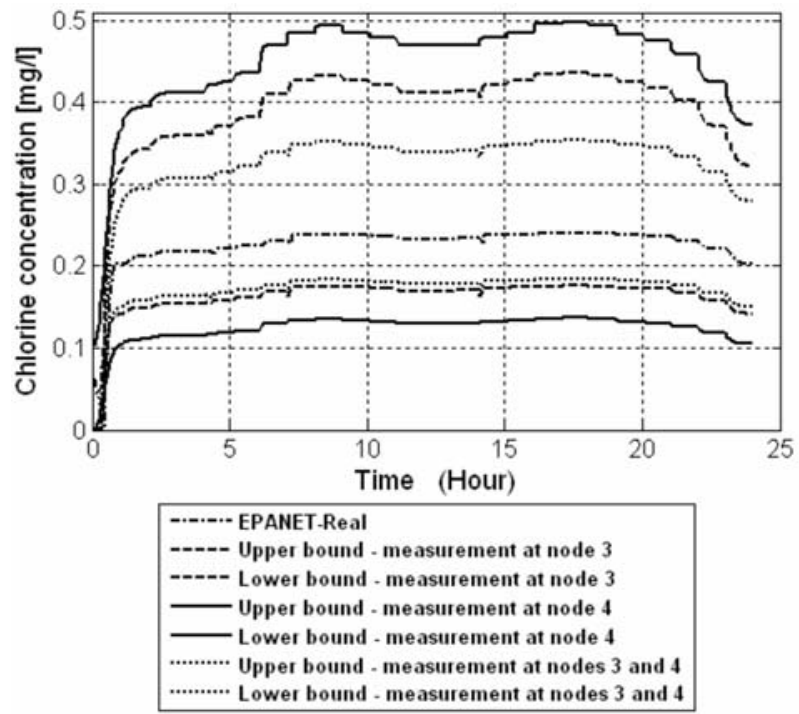

Fig. 23. Comparison of estimation performance at Node 5 for various locations and numbers of sensors.

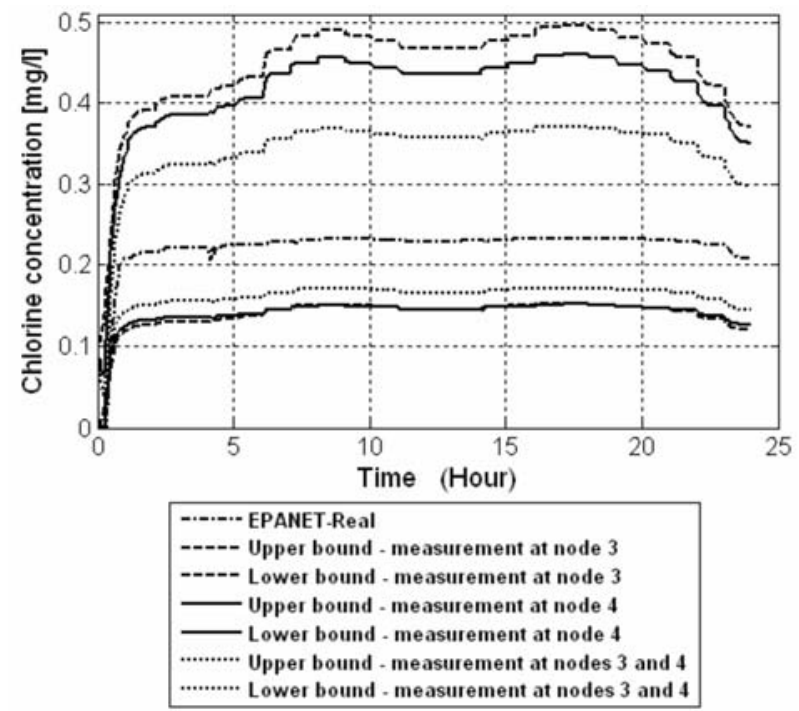

Fig. 24. Comparison of estimation performance at Node 6 for various locations and numbers of sensors.

\section{Conclusions and Future Work}

In the paper, the water quality model for estimation purposes has been derived and implemented in MATLAB. The interval observer was derived for a class of DWDSs and applied to an exemplary network. Estimation performance was examined for various locations of chlorine concentration sensors. The proposed observer produced promising results and the computational efficiency allows applying this observer to on-line water quality monitoring. Future work will focus on more complex network structures including tanks. Further research towards optimal use of the measurement information at the nodes with several connected pipes will be pursued. A rigours proof of the cor- 


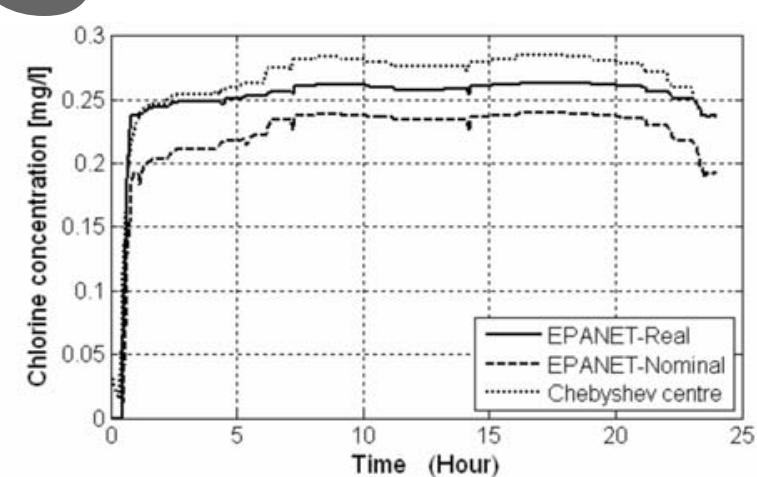

Fig. 25. Chlorine concentration point estimates and the real chlorine concentration at Node 5 .

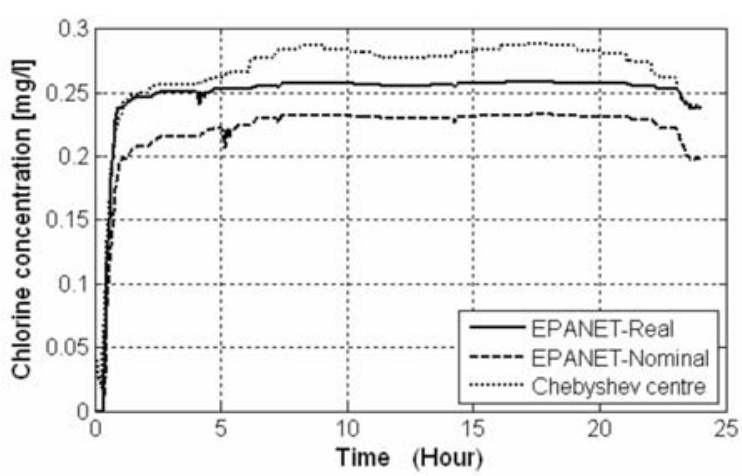

Fig. 26. Chlorine concentration point estimates and the real chlorine concentration at Node 6 .

rectness of the bounds produced by the interval observer is under way.

\section{Acknowledgment}

This work was supported by the Ministry of Science and Higher Education under Grant MiSterJa No. 4T11A-00825.

\section{References}

Al-Omari A.S. and Chaudhry M.H. (2001): Unsteady-state inverse chlorine modeling in pipe networks. - J. Hydr. Eng., Vol. 127, No. 8, pp. 669-677.

Alcaraz-González V., Harmand J., Rapaport A., Steyer J.P., González-Alvarez V. and Pelayo Ortiz C. (2004): Application of a robust interval observer to an anaerobic digestion process. - Proc. 10th IWA World Congress Anaerobic Digestion, AD10, Montreal, Canada, Vol. 1, pp. 337-342.

Boccelli D.L., Tryby M.E., Uber J.G. and Summers R.S. (2003): A reactive species model for chlorine decay and THM formation under rechlorination conditions. - Water Res., Vol. 37, No. 11, pp. 2654-2666.

Boulos P.F., Lansey K.E. and Karney K.E. (2004): Comprehensive Water Distribution Systems Analysis Handbook. Pasadena, CA: MWH Soft Inc.
Brdys M.A. and Ulanicki B. (1994): Operational Control of Water Systems: Structures, Algorithms and Applications. New York: Prentice Hall.

Brdys M.A. and Kang C.Y. (1994): Algorithms for state bounding in large-scale systems. - Int. J. Adapt. Contr. Signal Process., Vol. 8, No. 1, pp. 103-118.

Brdys M.A. and Chen K. (1995): Joint estimation of state and parameters in quantity models of water supply and distribution systems. - Automatisierungstechnik, Vol. 43, No. 2, pp. 77-84.

Brdys M.A. and Chen K. (1996): Joint estimation of states and parameters of integrated quantity and quality models of $d y$ namic water supply and distribution systems. - Proc. 13th IFAC World Congress, San Francisco, Vol. 1, pp. 73-78.

Chen K. (1997): Set membership estimation of state and parameters and operational control of integrated quantity and quality models of water supply and distribution systems. Ph.D. thesis, University of Birmingham, Birmingham, UK.

Clark R.M., Rossman L.A. and Wymer L.J. (1995): Modeling distribution system water quality: Regulatory implications. — J. Water Res. Plann. Manag., Vol. 121, No. 6, pp. 423428.

Duzinkiewicz K., Brdys M.A. and Chang T. (2005): Hierarchical model predictive control of integrated quality and quantity in drinking water distribution systems. - Urban Water J., Vol. 2, No. 2, pp. 125-137.

Duzinkiewicz K. (2006): Set membership estimation of parameters and variables in dynamic networks by recursive algorithms with a moving measurement window. - Int. J. Appl. Math. Comput. Sci., Vol. 16, No. 2, pp. 209-217.

Gouzé J.L., Rapaport A. and Hadj-Sadok M.Z. (2000): Interval observers for uncertain biological systems. - Ecol. Modell., Vol. 13, No. 1, pp. 45-56.

Grewal M.S. and Andrews A.P. (2001): Kalman Filtering: Theory and Practice, 2nd Ed.. - New York: Wiley.

Hadj-Sadok M.Z. and Gouzé J.L. (2001): Estimating of uncertain models of activated sludge process with interval observers. - J. Process Contr., Vol. 11, No. 3, pp. 299-310.

Harmand J. and Rapaport A. (2002): Interval observers for interconnected biotechnological systems. - Proc. 15th IFAC World Congress, Barcelona, Spain, (on CD-ROM).

Luenberger D.G. (1979): Introduction to Dynamic Systems: Theory, Models and Applications. — New York: Wiley.

Łangowski R. and Brdys M.A. (2006): Interval asymptotic estimator for chlorine monitoring in drinking water distribution systems. - Proc. 1st IFAC Workshop Applications of Large Scale Industrial Systems, Helsinki, Stockholm, (on CD-ROM).

Males R.M., Clark R.M., Wehrman P.J. and Gates W.E. (1985): Algorithm for mixing problems in water systems. - J. Hydr. Eng., Vol. 111, No. 2, pp. 206-219.

Males R.M., Grayman W.M. and Clark R.M. (1988): Modeling water quality in distribution systems. - J. Water Res. Plann. Manag., Vol. 114, No. 2, pp. 197-209. 
Milanese M., Norton J., Piet-Lahanier H. and Walter É. (1996): Bounding Approaches to System Identification. — New York: Plenum Press.

Mitchel A.R. and Griffiths D.F. (1980): The Finite Difference Method in Partial Differential Equations. - New York: Wiley.

Park K. and Kuo A.Y. (1999): A multi-step computation scheme: decoupling kinetic processes from physical transport in water quality models. - Water Res., Vol. 30, No. 10, pp. 2255-2264.

Rapaport A. and Dochain D. (2005): Interval observers for biochemical processes with uncertain kinetics and inputs. Math. Biosci., Vol. 193, No. 2, pp. 235-253.

Rossman L.A., Boulos P.F. and Altman T. (1993): Discrete volume element method for network water-quality models. J. Water Res. Plann. Manag., Vol. 119, No. 5, pp. 505-517.

Rossman L.A., Clark R.M. and Grayman W.M. (1994): Modeling chlorine residuals in drinking water distribution systems. - J. Env. Eng., Vol. 120, No. 4, pp. 803-820.

Rossman L.A. and Boulos P.F. (1996): Numerical methods for modeling water quality in distribution systems: a Comparison. - J. Water Res. Plann. Manag., Vol. 122, No. 2, pp. $137-146$.

Smith H.L. (1995): Monotone Dynamical Systems. An Introduction to the Theory of Competitive and Cooperative Systems. - Providence, Rhode Island: AMS.

\section{Appendices}

\section{A. Jacobian matrix}

We have $\boldsymbol{J}=\boldsymbol{A}\left(T_{H}\right)$. For our DWDS, the size of the Jacobian matrix for the first $T_{H}$ is $17 \times 17$ :

$$
\begin{aligned}
\boldsymbol{J} & =\boldsymbol{A}\left(T_{H}\right) \\
& =\left[\begin{array}{ccccc}
a_{1,1}\left(T_{H}\right) & \cdots & a_{1, i}\left(T_{H}\right) & \cdots & a_{1,17}\left(T_{H}\right) \\
\vdots & \ddots & & & \vdots \\
a_{j, 1}\left(T_{H}\right) & \cdots & a_{j, i}\left(T_{H}\right) & \cdots & a_{j, 17}\left(T_{H}\right) \\
\vdots & & & \ddots & \vdots \\
a_{17,1}\left(T_{H}\right) & \cdots & q_{17, i}\left(T_{H}\right) & \cdots & a_{17,17}\left(T_{H}\right)
\end{array}\right] .
\end{aligned}
$$

The diagonal elements are

$$
\begin{aligned}
& a_{1,1}\left(T_{H}\right)=a_{2,2}\left(T_{H}\right)=-\frac{v_{1 r}\left(T_{H}\right)}{\Delta l_{1 r}\left(T_{H}\right)}+k_{A}, \\
& a_{3,3}\left(T_{H}\right)=a_{4,4}\left(T_{H}\right)=a_{5,5}\left(T_{H}\right)=-\frac{v_{2 r}\left(T_{H}\right)}{\Delta l_{2 r}\left(T_{H}\right)}+k_{A}, \\
& a_{6,6}\left(T_{H}\right)=-\frac{v_{3 r}\left(T_{H}\right)}{\Delta l_{3 r}\left(T_{H}\right)}+k_{A},
\end{aligned}
$$

$$
\begin{aligned}
a_{7,7}\left(T_{H}\right) & =-\frac{v_{4 r}\left(T_{H}\right)}{\Delta l_{4 r}\left(T_{H}\right)}+k_{A}, \\
a_{8,8}\left(T_{H}\right) & =\cdots=a_{13,13}\left(T_{H}\right)=-\frac{v_{5 r}\left(T_{H}\right)}{\Delta l_{5 r}\left(T_{H}\right)}+k_{A}, \\
a_{14,14}\left(T_{H}\right) & =a_{15,15}\left(T_{H}\right)=-\frac{v_{6 r}\left(T_{H}\right)}{\Delta l_{6 r}\left(T_{H}\right)}+k_{A}, \\
a_{16,16}\left(T_{H}\right) & =a_{17,17}\left(T_{H}\right)=-\frac{v_{7 r}\left(T_{H}\right)}{\Delta l_{7 r}\left(T_{H}\right)}+k_{A} .
\end{aligned}
$$

Since $k_{A}$ is negative the diagonal elements shown above are also negative.

The non-zero off-diagonal elements are

$$
\begin{aligned}
a_{2,1}\left(T_{H}\right) & =\frac{v_{1 r}\left(T_{H}\right)}{\Delta l_{1 r}\left(T_{H}\right)}, \quad a_{6,2}\left(T_{H}\right)=\frac{v_{3 r}\left(T_{H}\right)}{\Delta l_{3 r}\left(T_{H}\right)}, \\
a_{7,5}\left(T_{H}\right) & =\frac{v_{4 r}\left(T_{H}\right)}{\Delta l_{4 r}\left(T_{H}\right)}, \quad a_{17,16}\left(T_{H}\right)=\frac{v_{7 r}\left(T_{H}\right)}{\Delta l_{7 r}\left(T_{H}\right)}, \\
a_{3,2}\left(T_{H}\right) & =a_{4,3}\left(T_{H}\right)=a_{5,4}\left(T_{H}\right)=\frac{v_{2 r}\left(T_{H}\right)}{\Delta l_{2 r}\left(T_{H}\right)}, \\
a_{14,6}\left(T_{H}\right) & =a_{15,14}\left(T_{H}\right)=\frac{v_{6 r}\left(T_{H}\right)}{\Delta l_{6 r}\left(T_{H}\right)} \\
a_{8,6}\left(T_{H}\right) & =a_{n, n-1}\left(T_{H}\right)=\frac{v_{5 r}\left(T_{H}\right)}{\Delta l_{5 r}\left(T_{H}\right)},
\end{aligned}
$$

where $n=9, \ldots, 13$,

$$
\begin{aligned}
a_{16,13}\left(T_{H}\right) & =\frac{v_{7 r}\left(T_{H}\right) Q_{5 r}\left(T_{H}\right)}{\Delta l_{7 r}\left(T_{H}\right)\left(Q_{4 r}\left(T_{H}\right)+Q_{5 r}\left(T_{H}\right)\right)}, \\
a_{16,7}\left(T_{H}\right) & =\frac{v_{7 r}\left(T_{H}\right) Q_{4 r}\left(T_{H}\right)}{\Delta l_{7 r}\left(T_{H}\right)\left(Q_{4 r}\left(T_{H}\right)+Q_{5 r}\left(T_{H}\right)\right)} .
\end{aligned}
$$

\section{B. Matrix $A_{11}^{+}\left(T_{H}\right)$}

In the exemplary DWDS considered, the matrix $\boldsymbol{A}_{11}^{+}\left(T_{H}\right)$ is sparse. Its size depends on the number of the estimated state variables, and for the first $T_{H}$ it is equal to $16 \times 16$. Hence

$$
\begin{aligned}
& \boldsymbol{A}_{11}^{+}\left(T_{H}\right) \\
& =\left[\begin{array}{ccccc}
a_{1,1}^{+}\left(T_{H}\right) & \cdots & a_{1, i}^{+}\left(T_{H}\right) & \cdots & a_{1,16}^{+}\left(T_{H}\right) \\
\vdots & \ddots & & & \vdots \\
a_{j, 1}^{+}\left(T_{H}\right) & \cdots & a_{j, i}^{+}\left(T_{H}\right) & \cdots & a_{j, 16}^{+}\left(T_{H}\right) \\
\vdots & & & \ddots & \vdots \\
a_{16,1}^{+}\left(T_{H}\right) & \cdots & a_{16, i}^{+}\left(T_{H}\right) & \cdots & a_{16,16}^{+}\left(T_{H}\right)
\end{array}\right] .
\end{aligned}
$$

The diagonal elements are

$$
\begin{aligned}
& a_{1,1}^{+}\left(T_{H}\right)=a_{2,2}^{+}\left(T_{H}\right)=-\frac{v_{1 r}^{-}\left(T_{H}\right)}{\Delta l_{1 r}\left(T_{H}\right)}+k_{A}, \\
& a_{3,3}^{+}\left(T_{H}\right)=a_{4,4}^{+}\left(T_{H}\right)=-\frac{v_{2 r}^{-}\left(T_{H}\right)}{\Delta l_{2 r}\left(T_{H}\right)}+k_{A}, \\
& a_{5,5}^{+}\left(T_{H}\right)=-\frac{v_{3 r}^{-}\left(T_{H}\right)}{\Delta l_{3 r}\left(T_{H}\right)}+k_{A}
\end{aligned}
$$




$$
\begin{aligned}
a_{6,6}^{+}\left(T_{H}\right) & =-\frac{v_{4 r}^{-}\left(T_{H}\right)}{\Delta l_{4 r}\left(T_{H}\right)}+k_{A}, \\
a_{7,7}^{+}\left(T_{H}\right) & =\cdots=a_{12,12}^{+}\left(T_{H}\right)=-\frac{v_{5 r}^{-}\left(T_{H}\right)}{\Delta l_{5 r}\left(T_{H}\right)}+k_{A}, \\
a_{13,13}^{+}\left(T_{H}\right) & =a_{14,14}^{+}\left(T_{H}\right)=-\frac{v_{6 r}^{-}\left(T_{H}\right)}{\Delta l_{6 r}\left(T_{H}\right)}+k_{A}, \\
a_{15,15}^{+}\left(T_{H}\right) & =a_{16,16}^{+}\left(T_{H}\right)=-\frac{v_{7 r}^{-}\left(T_{H}\right)}{\Delta l_{7 r}\left(T_{H}\right)}+k_{A} .
\end{aligned}
$$

Since $k_{A}$ is negative the diagonal elements shown above are also negative.

The non-zero off-diagonal elements are

$$
\begin{aligned}
& a_{2,1}^{+}\left(T_{H}\right)=\frac{v_{1 r}^{+}\left(T_{H}\right)}{\Delta l_{1 r}\left(T_{H}\right)}, \\
& a_{3,2}^{+}\left(T_{H}\right)=a_{4,3}^{+}\left(T_{H}\right)=\frac{v_{2 r}^{+}\left(T_{H}\right)}{\Delta l_{2 r}\left(T_{H}\right)}, \\
& a_{5,2}^{+}\left(T_{H}\right)=\frac{v_{3 r}^{+}\left(T_{H}\right)}{\Delta l_{3 r}\left(T_{H}\right)}, \\
& a_{7,5}^{+}\left(T_{H}\right)=a_{n, n-1}^{+}\left(T_{H}\right)=\frac{v_{5 r}^{+}\left(T_{H}\right)}{\Delta l_{5 r}\left(T_{H}\right)},
\end{aligned}
$$

where $n=8, \ldots, 12$,

$$
\begin{aligned}
& a_{13,5}^{+}\left(T_{H}\right)=a_{14,13}^{+}\left(T_{H}\right)=\frac{v_{6 r}^{+}\left(T_{H}\right)}{\Delta l_{6 r}\left(T_{H}\right)}, \\
& a_{16,15}^{+}\left(T_{H}\right)=\frac{v_{7 r}^{+}\left(T_{H}\right)}{\Delta l_{7 r}\left(T_{H}\right)}, \\
& a_{15,6}^{+}\left(T_{H}\right)=\frac{1}{\Delta l_{7 r}\left(T_{H}\right)} \\
& \quad \times \max _{v_{7 r}\left(T_{H}\right), Q_{4 r}\left(T_{H}\right), Q_{5 r}\left(T_{H}\right)}\left(\frac{v_{7 r}\left(T_{H}\right) Q_{4_{r}}\left(T_{H}\right)}{Q_{4 r}\left(T_{H}\right)+Q_{5 r}\left(T_{H}\right)}\right),
\end{aligned}
$$

$$
\begin{aligned}
& a_{15,12}^{+}\left(T_{H}\right)=\frac{1}{\Delta l_{7 r}\left(T_{H}\right)} \\
& \quad \times \max _{v_{7 r}\left(T_{H}\right), Q_{4 r}\left(T_{H}\right), Q_{5 r}\left(T_{H}\right)}\left(\frac{v_{7 r}\left(T_{H}\right) Q_{5 r}\left(T_{H}\right)}{Q_{4 r}\left(T_{H}\right)+Q_{5 r}\left(T_{H}\right)}\right),
\end{aligned}
$$

where $v_{l}^{+}\left(T_{H}\right)$ and $v_{l}^{-}\left(T_{H}\right)$ are respectively the upper and lower bounds on the linear water flow velocity at the $l$-th pipe over $T_{H}, l=1 r, \ldots, 7 r$, and $Q_{4 r}\left(T_{H}\right)$ and $Q_{5 r}\left(T_{H}\right)$ are the flow rate at the pipes $4 r$ and $5 r$ over $T_{H}$, respectively.

The matrix $\boldsymbol{A}_{11}^{-}\left(T_{H}\right)$ is determined in much the same way. For example,

$$
\begin{aligned}
& a_{15,6}^{-}\left(T_{H}\right)=\frac{1}{\Delta l_{7 r}\left(T_{H}\right)} \\
& \quad \times \min _{v_{7 r}\left(T_{H}\right), Q_{4 r}\left(T_{H}\right), Q_{5 r}\left(T_{H}\right)}\left(\frac{v_{7 r}\left(T_{H}\right) Q_{4_{r}}\left(T_{H}\right)}{Q_{4 r}\left(T_{H}\right)+Q_{5 r}\left(T_{H}\right)}\right), \\
& a_{15,12}^{-}\left(T_{H}\right)=\frac{1}{\Delta l_{7 r}\left(T_{H}\right)} \\
& \quad \times \min _{v_{7 r}\left(T_{H}\right), Q_{4 r}\left(T_{H}\right), Q_{5 r}\left(T_{H}\right)}\left(\frac{v_{7 r}\left(T_{H}\right) Q_{5 r}\left(T_{H}\right)}{Q_{4 r}\left(T_{H}\right)+Q_{5 r}\left(T_{H}\right)}\right) .
\end{aligned}
$$

Received: 11 October 2006 Revised: 15 May 2007 